\title{
A Two-DOF Bipedal Robot Utilizing the Reuleaux Triangle
}

\section{Drive Mechanism}

\author{
Jiteng Yang
}

Thesis submitted to the faculty of the Virginia Polytechnic Institute and State University in partial fulfillment of the requirements for the degree of

\author{
Master of Science \\ In \\ Mechanical Engineering \\ Pinhas Ben-Tzvi, Chair \\ Steve Southward \\ Tomonari Furukawa
}

November 16, 2018

Blacksburg, Virginia

Keywords: Bipedal Robot, Reduced DOF, Reuleaux Triangle

Copyright $\odot 2018$ 


\title{
A Two-DOF Bipedal Robot Utilizing the Reuleaux Triangle Drive Mechanism
}

\author{
by Jiteng Yang
}

\begin{abstract}
This thesis explores the field of legged robots with reduced degree-of-freedom (DOF) leg mechanisms. Multi-legged robots have drawn interest among researchers due to their high level of adaptability on unstructured terrains. However, conventional legged robots require multiple degrees of freedom and each additional degree of freedom increases the overall weight and complexity of the system. Additionally, the complexity of the control algorithms must be increased to provide mobility, stabilization, and maneuvering. Normally, robotic legs are designed with at least three degrees of freedom resulting in complex articulated mechanisms, which limits the applicability of such robots in real-world applications. However, reduced DOF leg mechanisms come with reduced tasking capabilities, such as maintaining constant body height and velocity during locomotion.

To address some of the challenges, this thesis proposes a novel bipedal robot with reduced DOF leg mechanisms. The proposed leg mechanism utilizes the Reuleaux triangle to generate the foot trajectory to achieve a constant body height during locomotion while maintaining a constant velocity. By using a differential drive, the robot is also capable of steering. In addition to the analytical results of the trajectory profile of each leg, the thesis provides a trajectory function of the Reuelaux triangle cam with respect to time such that the robot can maintain a constant velocity and constant body height during walking. An experimental prototype of the bipedal robot was integrated and experiments were conducted to evaluate the walking capability of the robot. Ongoing future work of the proposed design is also outlined in the thesis.
\end{abstract}




\section{A Two-DOF Bipedal Robot Utilizing the Reuleaux Triangle Drive Mechanism by Jiteng Yang}

\section{GENERAL AUDIENCE ABSTRACT}

Bipedal robots are a type of legged robots that use two legs to move. Legs require multiple degrees of freedom to provide propulsion, stabilization, and maneuvering. Additional degrees of freedom of the leg result in a heavier robot, more complex control method, and more energy consumption. However, reduced degree of freedom legs result in a tradeoff between certain tasking capabilities for easier controls and lower energy consumption.

As an attempt to overcome these challenges, this thesis presents a robot design with a reduced degree of freedom leg mechanism. The design of the mechanism is described in detail with its preliminary analysis. In addition, this thesis presents experimental validation with the robot which validates that the robot is capable of moving with constant body height at constant velocity while being of capable of steering. The thesis concludes with a discussion of the future work. 


\section{ACKNOWLEDGEMENTS}

Firstly, I would like to thank my family and friends, especially my mother Zhenhuan Wu, my father Guo'an Yang, my friends Elliot Fairhurst and Scott Farmer for all their love and support throughout my academic studies. I would also like to thank Qiao Chen for her support and encouragement while I am overseas. They helped me remain strong-minded throughout my time in college, despite the many challenges and hardships I faced along the way.

This thesis will cover the work I accomplished for my Master's degree. My research would not have been successful without the help of many people. I would like to thank my advisor, Professor Pinhas Ben-Tzvi for giving me a chance to expand my knowledge by becoming a part of his Robotics and Mechatronics Lab at Virginia Tech. Besides being a supportive advisor, his encouraging words and enthusiastic attitude pushed me to perform at the highest quality. In addition to having an understanding advisor, I am grateful for having the opportunity to be surrounded by knowledgeable people in our lab. I am thankful for Wael Saab, who served as my senior mentor in the lab, Zhoubao Pang and Hongxu Guo for their assist in constructing and programming the robot. With his assistance throughout my research work, I was able to achieve great progress in completing my research goals. 


\section{TABLE OF CONTENTS}

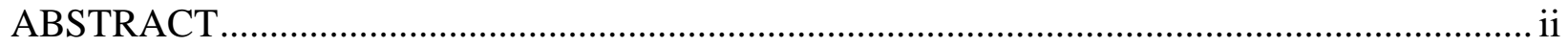

GENERAL AUDIENCE ABSTRACT ................................................................................. iii

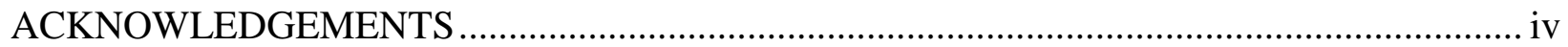

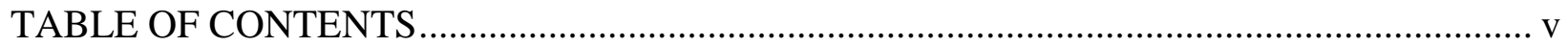

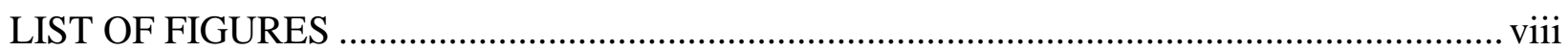

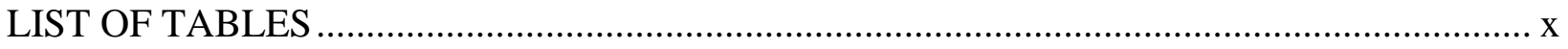

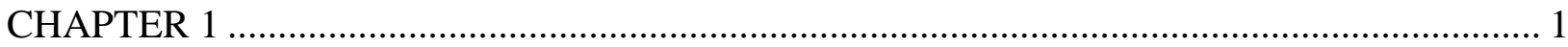

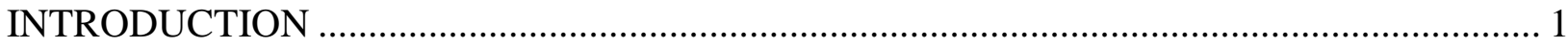

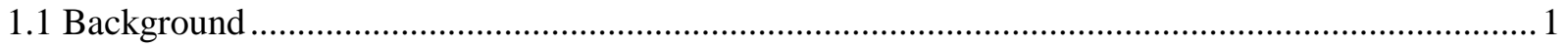

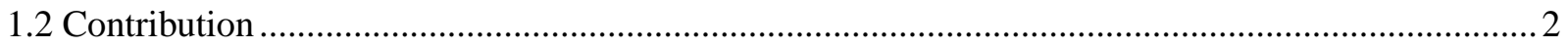

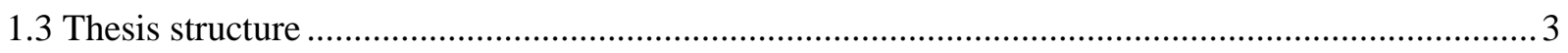

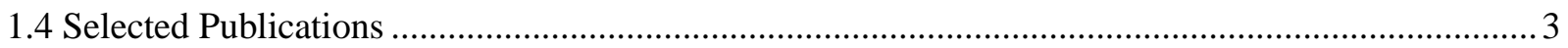

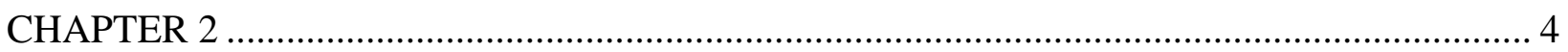

LITERATURE REVIEW AND MOTIVATION ............................................................. 4

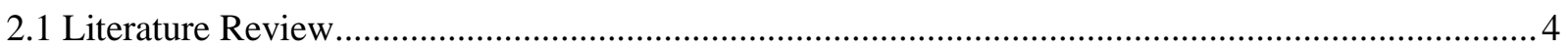

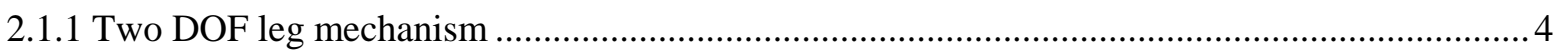

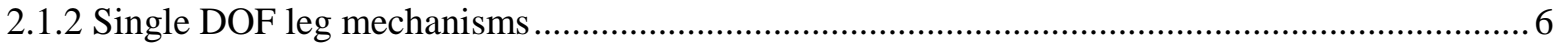

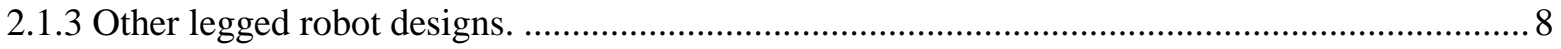

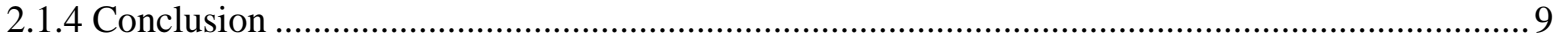

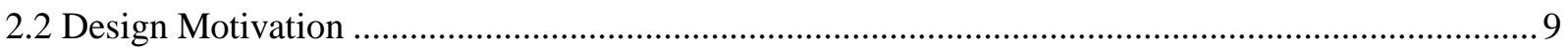

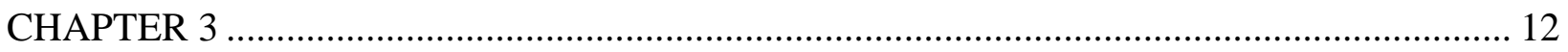

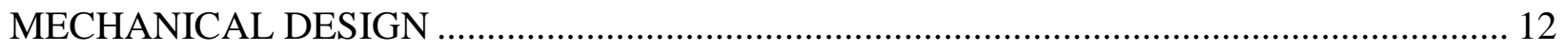

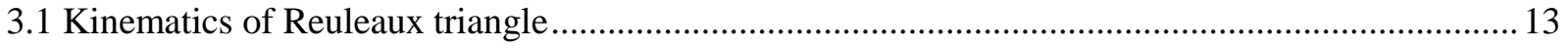




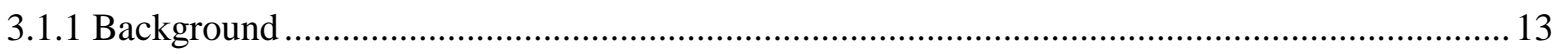

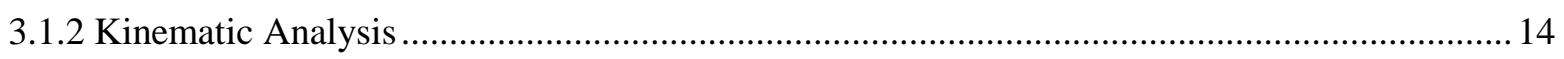

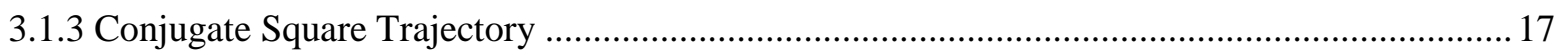

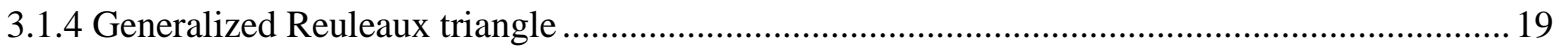

3.1.5 Friction Between the Cam and the Cam-follower................................................................21

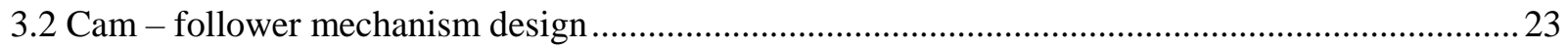

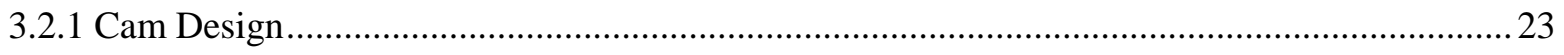

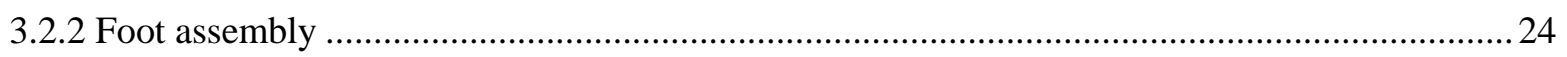

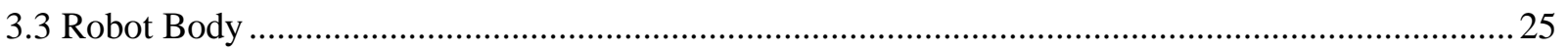

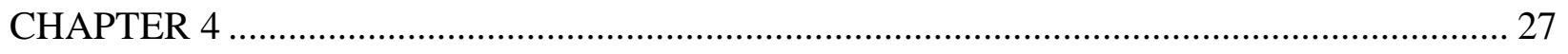

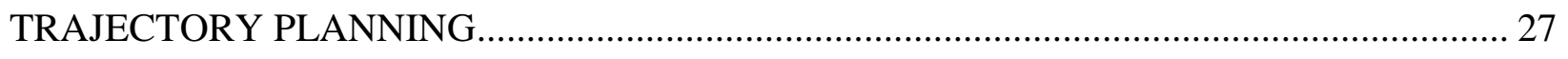

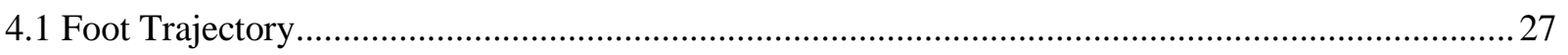

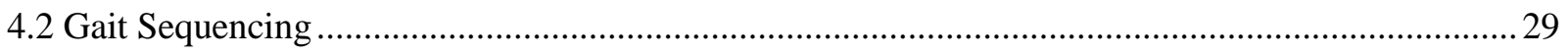

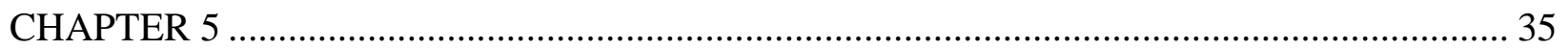

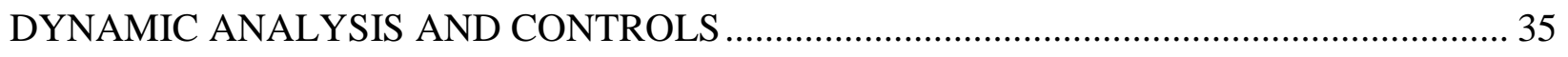

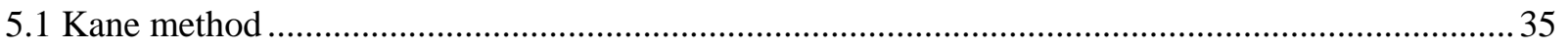

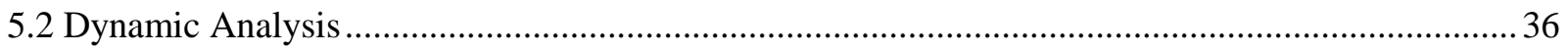

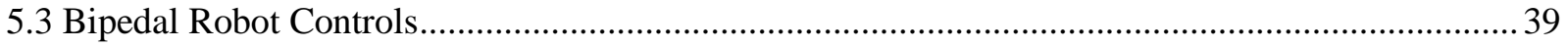

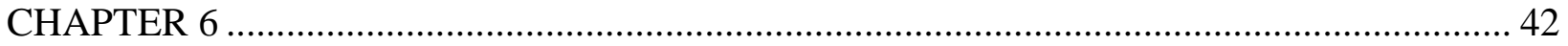

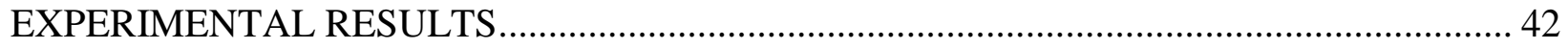

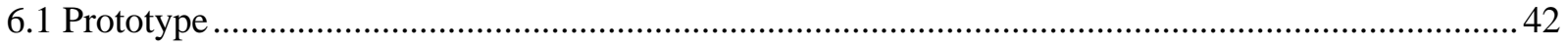

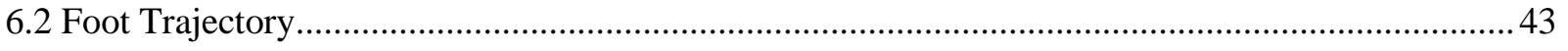

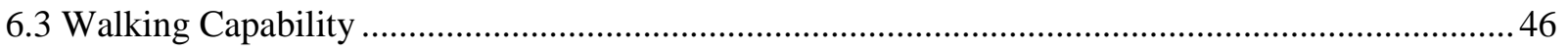

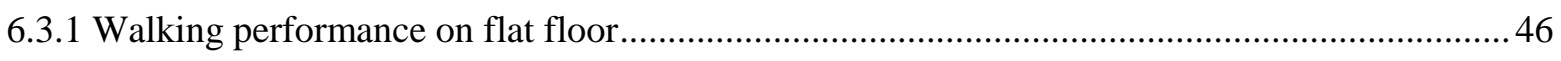

6.3.2 Walking performance on rough terrains ...................................................................... 50

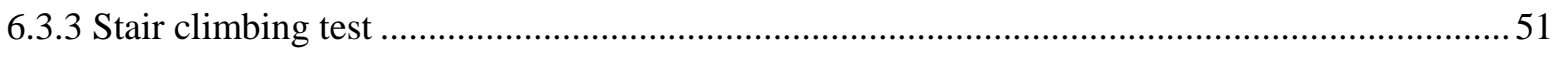

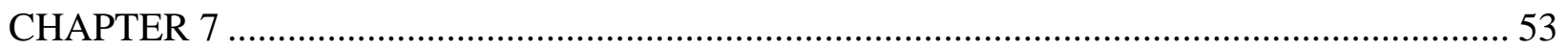




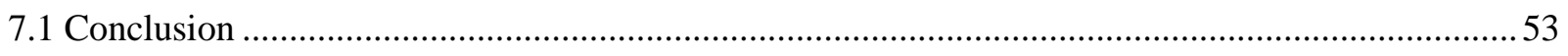

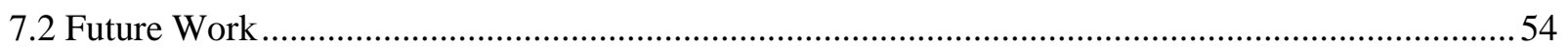

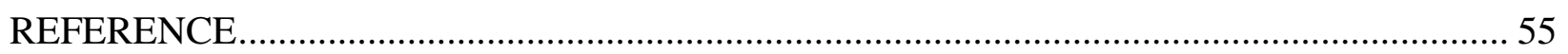




\section{LIST OF FIGURES}

Figure 2.1: First generation Robotic Modular Leg $[10,11]$.............................................. 4

Figure 2.2: Centipede-like robot proposed by Torige [12] ............................................ 5

Figure 2.3: Centipede-like robot proposed by Hoffman [13] ........................................... 5

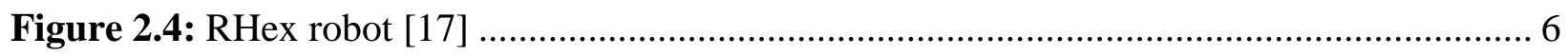

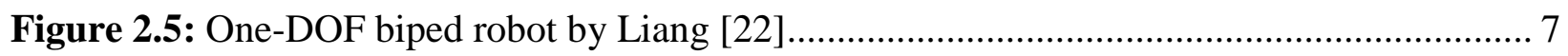

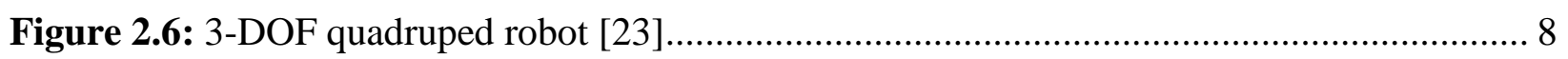

Figure 3.1: Schematic diagram of the RML-V2 ....................................................... 12

Figure 3.2: Schematic of Reuleaux triangle and its conjugate square .................................. 14

Figure 3.3: Trajectory profiles of point $G$ normalized for various offset values $\rho \ldots \ldots \ldots \ldots \ldots \ldots \ldots . . . . . . .18$

Figure 3.4: Generalized Reuleaux triangle ................................................................ 20

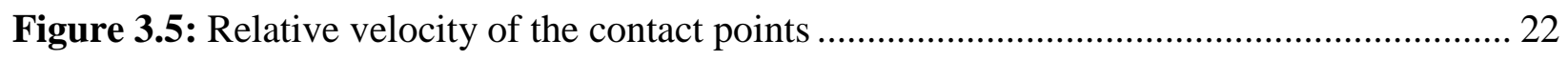

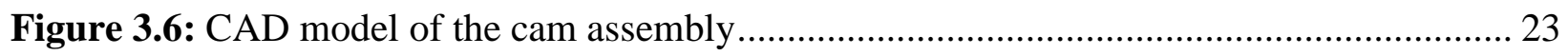

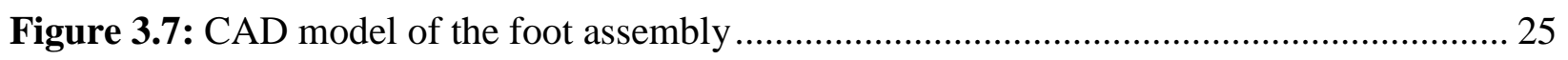

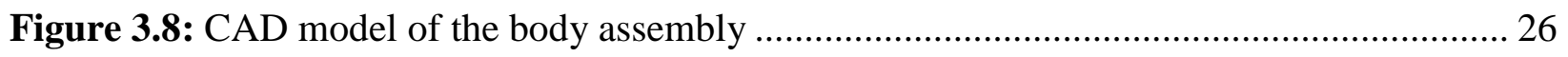

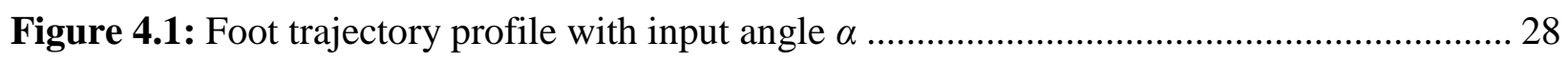

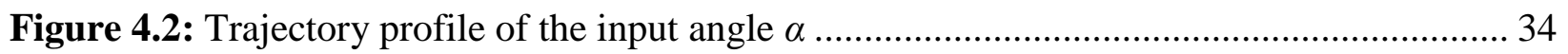

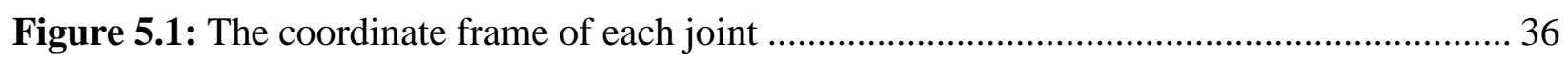

Figure 5.2: The control block diagram of the robot....................................................... 39

Figure 5.3: Simulated PI controller tracking results ..................................................... 40

Figure 5.4: Calculated torque on both motors …....................................................... 41

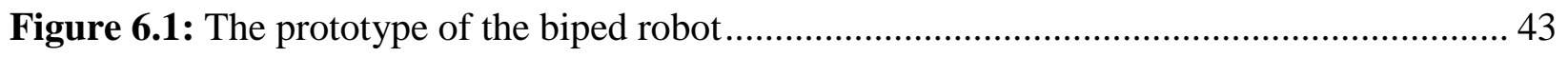

Figure 6.2: Experimental and calculated foot trajectory.................................................. 44 
Figure 6.3: $x$ error and $y$ error of experimental results versus theoretical results

Figure 6.4: Straight walking prototype body $x$ and $y$ displacement.

Figure 6.5: Biped robot demonstrating forward locomotion (A-F), and differential turning (G-L)

Figure 6.6: Experiment setup for rough terrain walking performance test.......................... 51

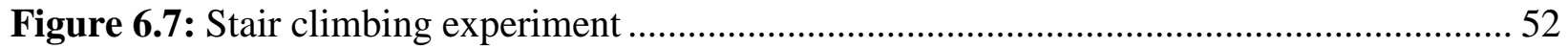




\section{LIST OF TABLES}

Table 2.1: Comparison of different leg mechanisms .................................................... 10

Table 3.1: States of Reuleaux Triangle Rotation .................................................................. 15

Table 5.1: The Relation of the Coordinate Frames ............................................................ 37 


\section{CHAPTER 1}

\section{INTRODUCTION}

\subsection{Background}

The recent increase in the interest in robotic bipedal locomotion has inspired the development of a novel type of bipedal leg mechanism with potential application in all bipedal robotic systems. A traditional bipedal robot requires a behavior level controller that must generate high level commands, with a lower level controller that generates joint commands [1] and multiple active DOF leg mechanism. We aim to circumvent the complexity in the design and controls of the traditional biped legged robot by proposing a reduced degree-of-freedom (DOF) leg mechanism. The objective of this thesis is to develop a robot that can simplify the control of the biped robot without sacrificing its walking capabilities on flat surfaces, and to reduce the energy consumption of the robot. The proposed design in this thesis should also provide a stable robotic platform decoupled from primary manipulators to aid in the future robotics research.

Conventional robotic legs on robots resemble biological legs which rely on multiple active DOFs to enhance locomotion and tasking capabilities [2]. However, additional DOFs require additional actuators, which results in an increase in the overall weight, energy consumption, and the complexity of the control algorithms to simultaneously provide propulsion, stabilization, and maneuvering [3]. Therefore, there are very few robots that have been successfully implemented in real life applications. Such examples include the ANYmal quadruped [4] and Adaptive Suspension Vehicle [5]. 
A majority of existing legged robots are inspired by nature. Animals evolved to adapt to their natural habitat and this serves as a reference for researchers to develop their legged robots. These robots utilize multiple DOFs to position their single point of contact foot. The typical walking robots use three DOFs for each leg [6,7], which are traditionally allocated to the hip abduction/adduction and hip and knee extension/flexion. The hip abduction/adduction enables turning, while hip and knee extension/flexion enables planar walking. Therefore, a multi-legged robot, with $n$ pairs of legs, normally requires $6 n$ actuators. If flat feet are implemented, a more stable gait can be achieved due to a larger support polygon, however, additional DOFs are required to control the orientation of the foot [8].

The robot proposed in this thesis was designed to address the challenges of highly articulated legs by utilizing reduced DOF leg mechanisms.

\subsection{Contribution}

In this thesis, we present the design and integration of a bipedal robot design which is composed of two single DOF Robotic Modular Leg Mechanisms (RML-V2). A prototype was designed and experimental testing was conducted to evaluate the system performance. The work of this thesis presents the following contributions to the field of reduced DOF robots:

1. A novel implementation of the Reuleaux triangle cam-follower mechanism to actuate a robotic leg as a single DOF

2. A robotic leg mechanism with quasi-static stability inherent in its design

3. A bipedal robotic system that is capable of walking with constant body height

4. Full control of forward/backward locomotion with complete turning ability

5. Provide functions of the Reuleaux triangle conjugate square trajectory with respect to the offset of the pivot point from the Reuelaux triangle centroid. 


\subsection{Thesis structure}

The rest of the thesis is organized as follows:

Chapter 1: Presents the introduction and main contribution of this thesis

Chapter 2: A comprehensive literature review on legged robots and discussions of these state-ofthe-art systems

Chapter 3: Presents the mechanical design of the reduced DOF leg mechanism and the kinematic properties of the Reuleaux triangle cam-follower mechanism

Chapter 4: Presents the analysis and optimization of the foot trajectory profiles and functions of the Reuleaux triangle cam rotating angle with respect to time.

Chapter 5: Presents a brief dynamic analysis of the system and the control solution of the robot such that the Reuleaux triangle could follow the given trajectory

Chapter 6: Presents the integrated prototype and experimental validation of the mechanical design and results of the waking capability evaluation tests

Chapter 7: Conclude the thesis by providing a summary of the work and a discussion about future work.

\subsection{Selected Publications}

Disclosure: Content from this publication was used throughout the thesis.

\section{Journal Paper}

J. Yang, W. Saab, and P. Ben-Tzvi, “A Two-DOF Bipedal Robot Utilizing the Reuleaux Triangle Drive Mechanism", ASME Journal of Mechanisms and Robotics, Submitted, September 2018. 


\section{CHAPTER 2}

\section{LITERATURE REVIEW AND MOTIVATION}

\subsection{Literature Review}

The reduced DOF leg mechanism utilizes two or fewer active DOFs to achieve locomotion capabilities. However, a reduced DOF results in fewer walking capabilities, such as maintaining constant body height or orientation during walking maneuvers [9]. An overview of previously proposed leg mechanisms and legged robots is provided below, with a particular focus on design and functionality.

\subsubsection{Two DOF leg mechanism}

The first generation Robotic Modular Legs (RM Leg) [10, 11] and centipede like robots [12] [13] have two active DOFs per leg. The first generation RM Leg mechanism uses double four-bar mechanisms connected in series to restrict the orientation of the foot (Figure 2.1). The foot trajectory shows a straight line support phase, which is when the foot is in contact with the ground.
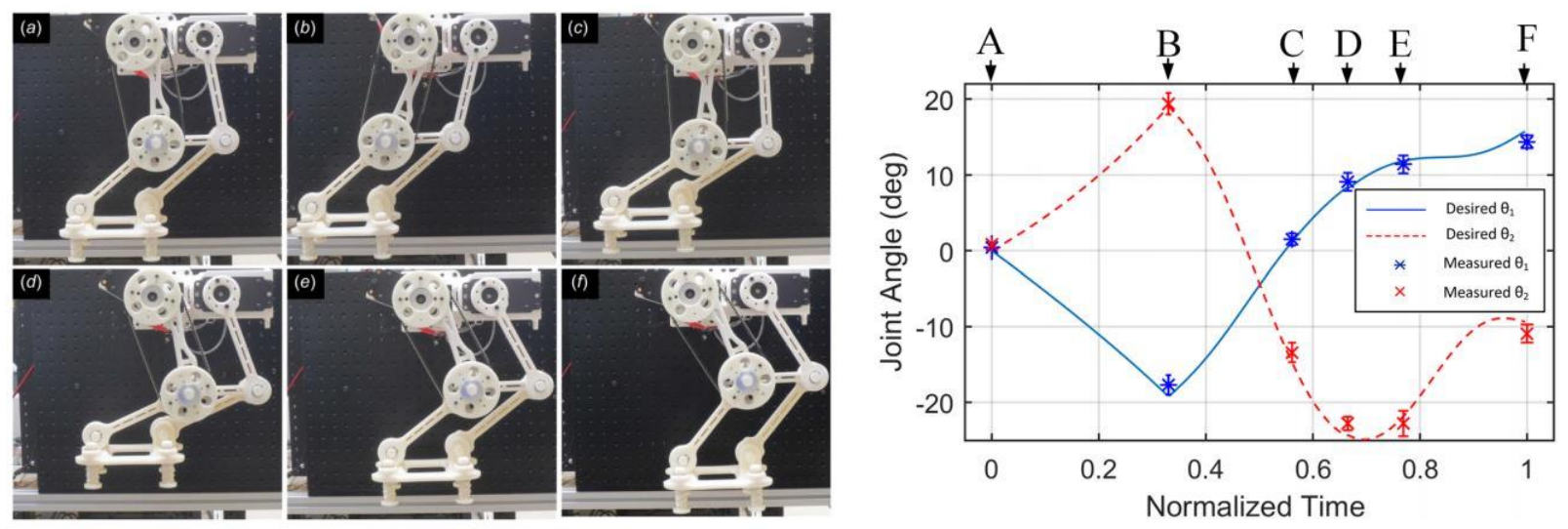

Figure 2.1: First generation Robotic Modular Leg [10, 11] 
The centipede like robot has multiple segments and each segment has two legs. One DOF of the leg is used to raise the robot leg and the other DOF is used to swing the leg back and forth to facilitate locomotion. Torige's design (Figure 2.2) [12] uses a revolute joint to raise the leg and a prismatic joint to swing the leg, while Hoffman's design (Figure 2.3) [13] used two revolute joints powered by piezoelectric bimorph actuators to raise and swing the legs.
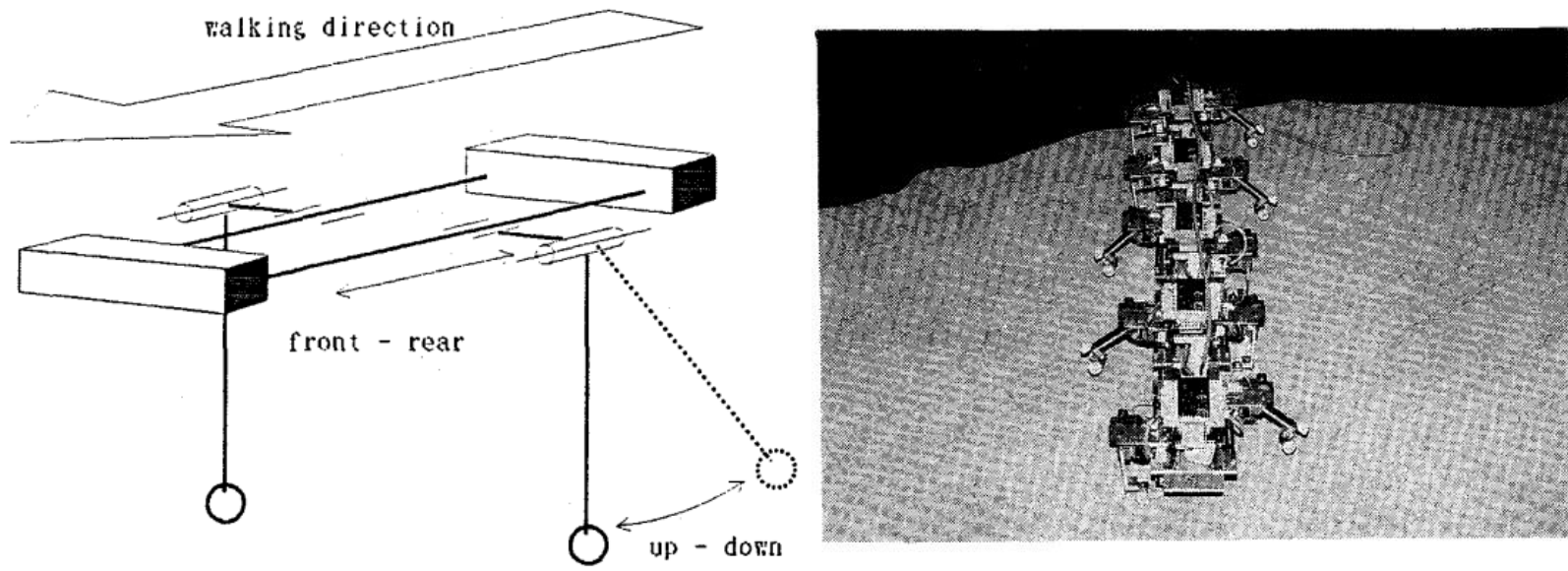

Figure 2.2: Centipede-like robot proposed by Torige [12]
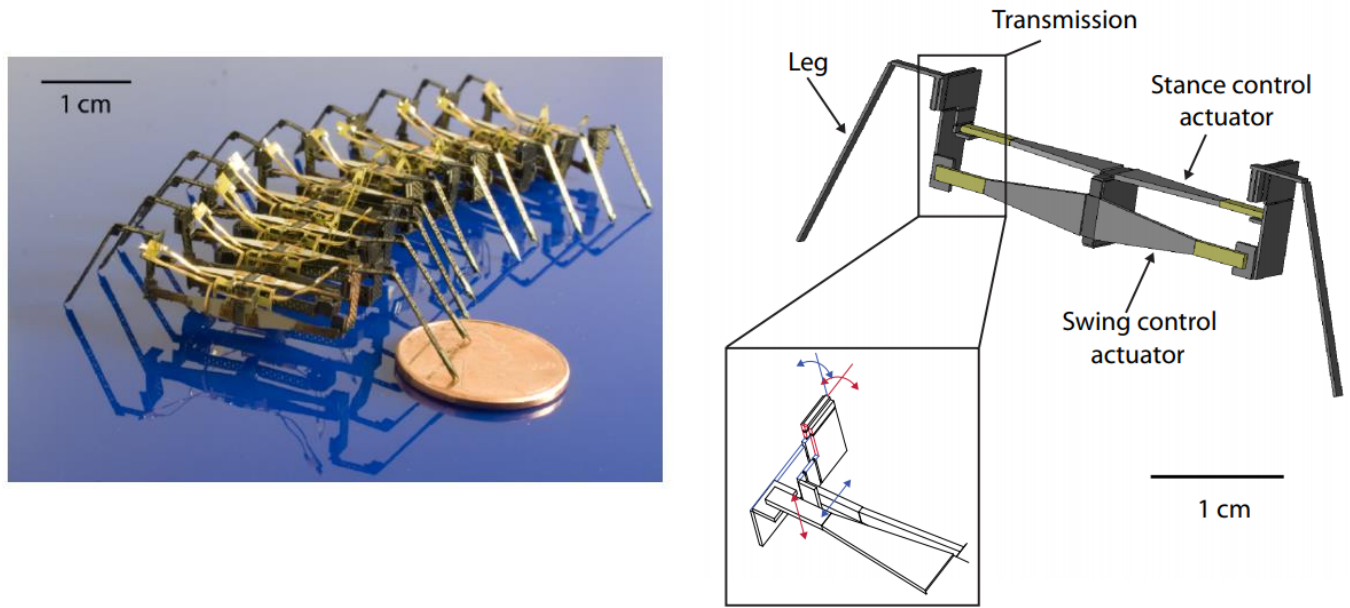

Figure 2.3: Centipede-like robot proposed by Hoffman [13]

Unfortunately, for the RM Leg to achieve a straight line support phase, the actuators need to be sequenced. For centipede like robots, multiple body segments of the robot are required to 
achieve a stable stance. For Hoffman's centipede like robot, by using revolute joint to swing the robot, each segment is unable to maintain a constant orientation during locomotion.

Adachi [14] proposed a leg mechanism utilizing a revolute joint and a prismatic joint. A coupled actuation of the revolute joint and prismatic joint allows the foot mechanism to achieve a straight line support phase.

Lovasz [15], inspired by single DOF Jansen leg mechanisms [16], proposed that using a 5-link belt mechanism with two DOFs can be adapted to generate a similar foot trajectory as Jansen leg mechanisms. The mechanism avoids singularities during locomotion and can be used to follow Jansen leg foot trajectory or achieve similar trajectories with larger step height to overcome obstacles.

\subsubsection{Single DOF leg mechanisms}

RHex hexapod robot [17] uses six single DOF legs to drive the robot. The robot uses continuous rotation of its " $\mathrm{C}$ " shaped leg to propel the robot and use differential drive to enable steering. The foot trajectory of each leg is a circle and therefore, the robot was unable to maintain a constant body height during locomotion.

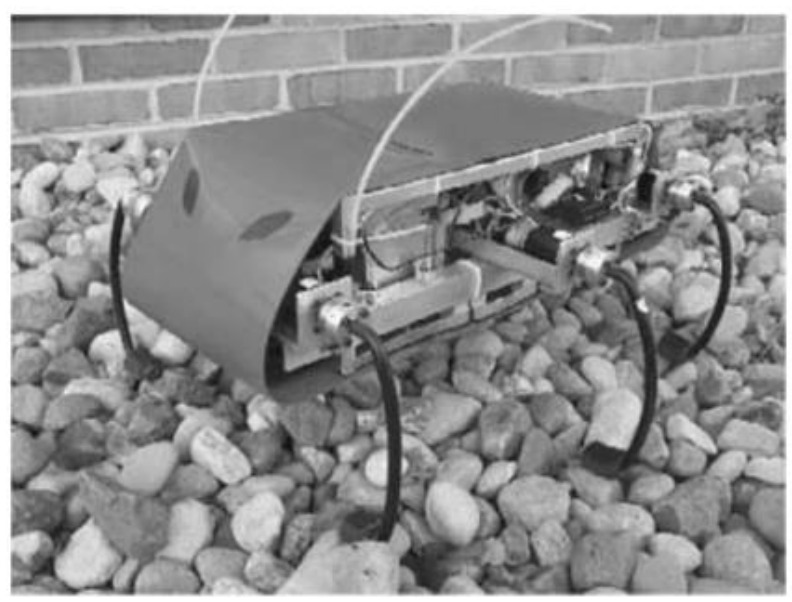

Figure 2.4: RHex robot [17] 
Other single DOF leg mechanisms involve utilization of single crank driving mechanisms. Jansen leg mechanisms produce an approximate straight line support phase for walking. The leg mechanism is developed by Theq Jansen and is used on the Strandbeests that move about on the beach powered only by the wind [16]. Funabashi [18] performed basic experiments on a hexapod walking machine with an approximate straight line support phase. In his research, he pointed out that the ankle path generator should be selected from among planar 4-bar and 6-bar mechanisms with only revolute joints. Kaneko [6] experimented with a hexapod walking machine which uses approximate straight-line linkage mechanisms as legs. Tavolieri [19] conducted an analysis and designed a leg mechanism in 2006 and 2008. Klann's mechanism was used on mechanical spider proposed by Lokhande [20] in 2013. The mechanical spider uses four pairs of Klann mechanism legs to drive the robot. The robot could achieve an approximate constant body height during locomotion. However, the one DOF foot limits the motion of the robot and therefore, the robot can only move laterally and cannot steer. Kamidi [21] proposed a planar leg design for quadruped robot that moves at high speed. The leg mechanism uses single point contact with the ground. The mechanism uses a continuously rotating link to drive a six-bar mechanism.

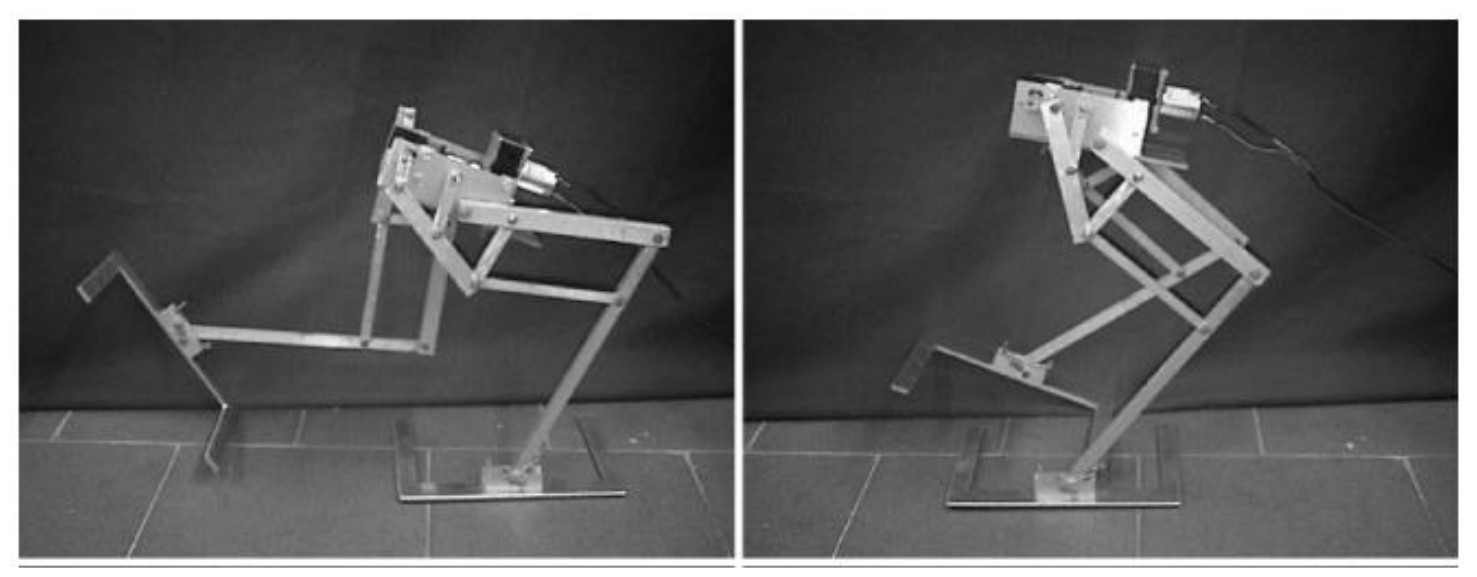

Figure 2.5: One-DOF biped robot by Liang [22] 
The most iconic biped robot design with single DOF leg mechanism was proposed by Liang [22]. It utilizes a one DOF pantograph leg mechanism to achieve locomotion of the robot. Even though the foot trajectory of the leg design demonstrates a straight-line segment, the robot however failed to achieve a constant body height during locomotion. The experimental results in literature indicate a fluctuation in the vertical acceleration of the body, which Liang pointed out was caused by the up and down motion of the body during the locomotion. It is worth noting the design presented in this thesis prioritizes the avoidance of this type of vertical oscillation.

\subsubsection{Other legged robot designs.}

Yoenda [23] proposed a three DOF quadruped with three active DOF. This robot consists of front and rear sections. Passive joints are used to connect "U" shaped legs to the body. A rotation joint controls the rotation of two sections and, using a transmission system, controls the width of the "U" shaped legs. Two prismatic joints control the translational motion of the two sections. The motion of each segment coupled with the passive motion of the leg allows the robot to move forward. The robot can move at a velocity of $40 \mathrm{~mm} / \mathrm{s}$.

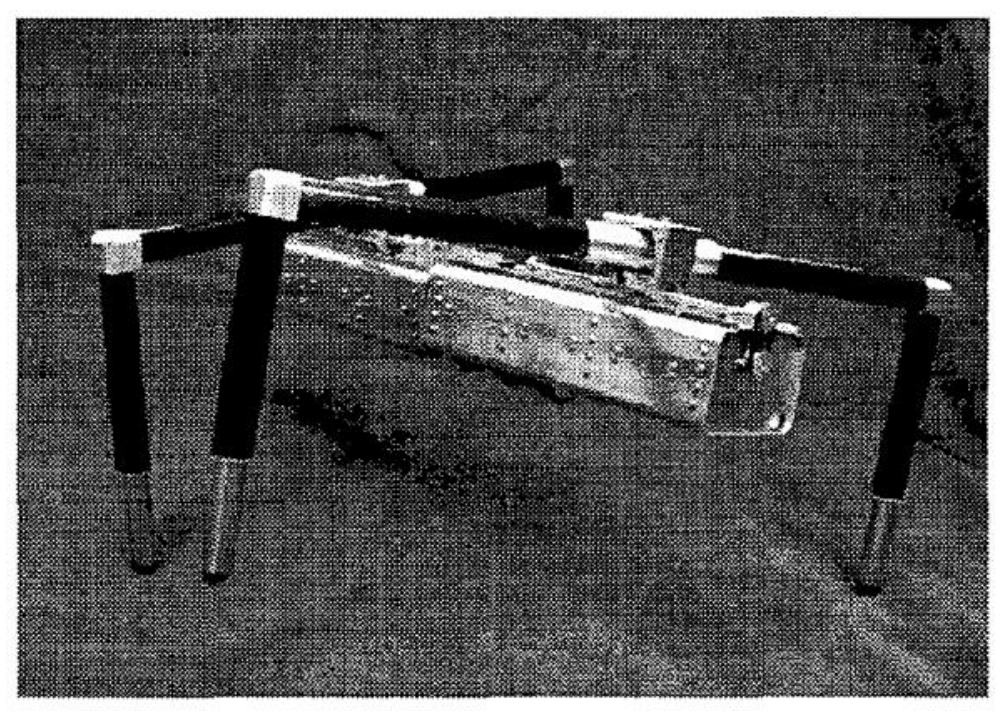

Figure 2.6: 3-DOF quadruped robot [23] 
The motion of the robot relies on the rotation and extension/contraction of the body. There are two configurations of the robot in which the robot is stable and each configuration can be switched to another configuration when a disturbance is applied. Furthermore, since the length of the leg remains constant, the body height changes during locomotion which induces instability and greater energy consumption.

\subsubsection{Conclusion}

The primary goal for the reduced leg mechanism is to achieve walking capability with the robot. However, tradeoffs can affect the walking capabilities of the robot and cause instability; these can be solved by utilizing multiple legs. For bipedal robots, a larger foot can be used to prevent the robot from tipping over when one foot is in support phase and the other is in swing phase. The body height fluctuation during the locomotion can create instability and can affect walking performance.

\subsection{Design Motivation}

A literature review on the reduced DOF leg mechanism was given in the previous chapter, multiple approaches for the legged robots with reduced DOF leg mechanisms were discussed and the comparison of different leg mechanisms are presented in Table 2.1. The analysis highlighted the advantages and disadvantages of several state-of-the-art designs, identifying common problems presented in the design. This chapter further discusses these common design problems and presents the goals of the proposed mechanism for a new biped legged robot design.

Although reduced DOF leg mechanisms enable the construction of legged robots with simpler mechanical design and a reduced level of complexity, the decreased articulation may hinder the robot's walking performance. As mentioned in the literature review, although X-Rhex [13] and 
Liang's bipedal robot [18] are capable of walking forward, the robot body height and orientation fluctuate during a walking gait, which is undesirable.

Table 2.1: Comparison of different leg mechanisms

\begin{tabular}{ccccc}
\hline & $\begin{array}{c}\text { RM Leg } \\
{[10,11]}\end{array}$ & $\begin{array}{c}\text { Centipede-like robot } \\
{[12][13]}\end{array}$ & RHex [17] & One-DOF robot [22] \\
\hline DOFs & 2 & 2 & 1 & 1 \\
\hline $\begin{array}{c}\text { Support phase } \\
\text { trajectory }\end{array}$ & Straight line & $\begin{array}{c}\text { Straight line/Horizontal } \\
\text { curve }\end{array}$ & $\begin{array}{c}\text { Vertical } \\
\text { circular curve }\end{array}$ & Spline \\
\hline Body height & Constant & Constant & Fluctuate & Fluctuate \\
\hline $\begin{array}{c}\text { Body } \\
\text { orientation }\end{array}$ & Constant & Constant/Fluctuate & Constant & Constant \\
\hline
\end{tabular}

The fluctuation of the body height may induce instability and require additional energy to raise the body vertically against gravity and the fluctuation in body orientation may cause the robot to move off course.

Therefore, five design criteria from reference [8] were presented:

1. Maintaining quasi-static stability during locomotion

2. Maintaining a constant body height and body orientation

3. Maintaining constant velocity during locomotion

4. Moving forward

5. Ability to steer the robot

Criteria 1 and 2 are required to maintain a stable robotic platform in static configuration and during a walking gait. These criteria improve the energy efficiency of the system since the legs are not required to raise or lower the body cyclically. Criterion 2 also ensures a sufficient margin of stability. Criterion 3 requires the robot to move with a constant velocity which eliminates the inertial forces acting on the robot body improving the stability as the center-of-mass of the robot 
is always projected into the support polygon. Criteria 4 and 5 ensure the locomotion capabilities required to enable the robot to move forward and be steered in any desired position and direction. 


\section{CHAPTER 3}

\section{MECHANICAL DESIGN}

The schematic diagram of the RML-V2 is presented in Figure 3.1. The leg is composed of double four-bar mechanism parallelograms connected in series and a Reuleaux triangle camfollower mechanism. The two four-bar mechanisms restrict the rotational motion of the foot such that it maintains a constant angular orientation with respect to the body and therefore the need of an additional motor to at the ankle is eliminated. The RML-V2 is a single DOF mechanism that utilizes a generalized Reuleaux triangle cam-follower system. The foot of the leg is the conjugate square of the generalized Reuleaux triangle.
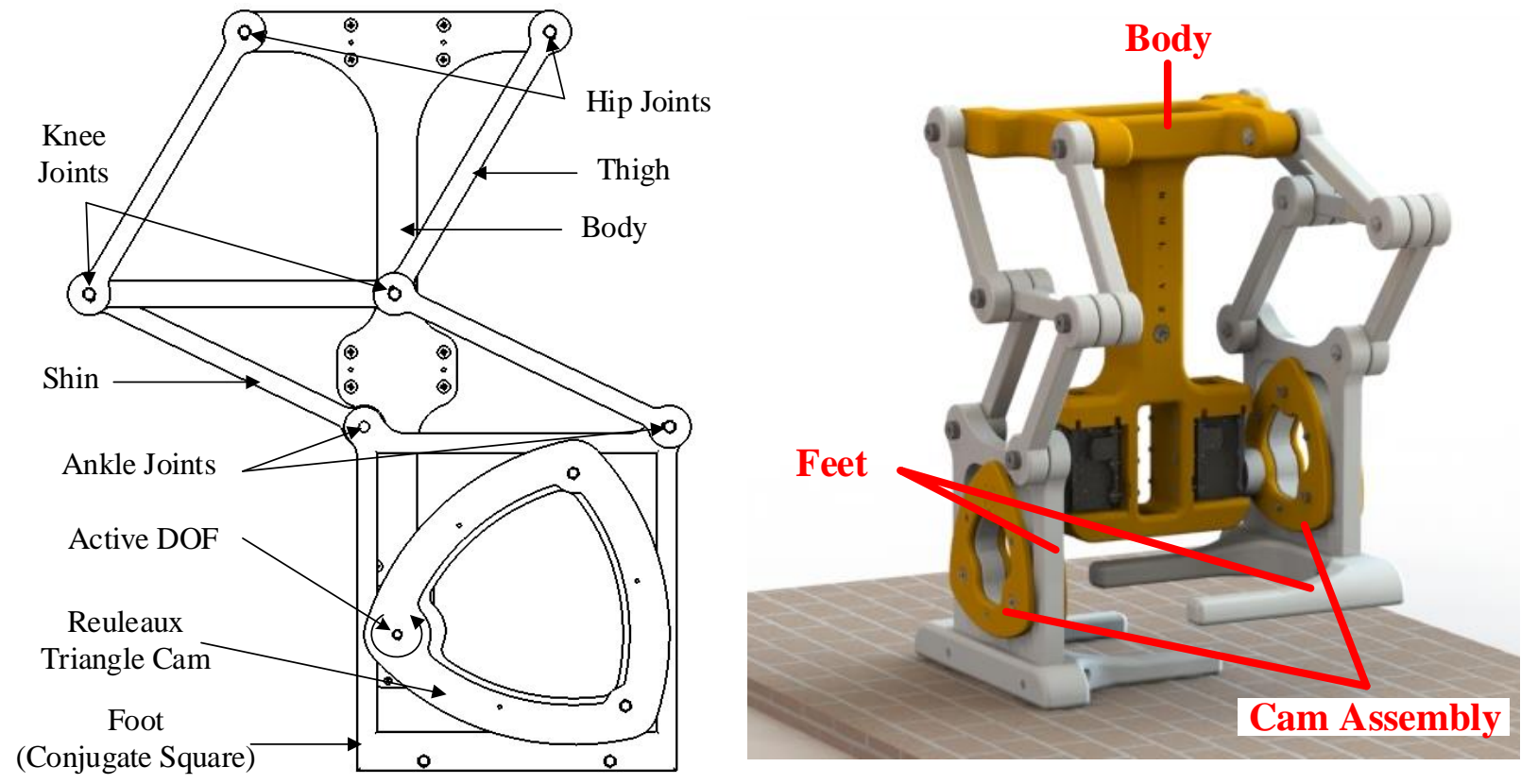

Figure 3.1: Schematic diagram of the RML-V2

In this design, the Reuleaux triangle is set to rotate with an offset from its centroid to produce a desirable foot trajectory. 


\subsection{Kinematics of Reuleaux triangle}

\subsubsection{Background}

The Reuleaux triangle is named after Franz Reuleaux who presented a qualitative study on this geometry in his book "Kinematics of Machinery: outlines of a theory of machines" [24]. It is constructed by drawing arcs from each vertex of the equilateral triangle with a radius equivalent to the length of the triangle. Therefore, it has a constant breadth and can rotate inside a square while maintaining contact on all sides of the square.

The first appearance of Reuleaux triangles was in a work by Leonardo da Vinci circa 1514, where he projected map of the Earth onto a circle using eight pairs of triangular-shaped octants which consists of an equilateral triangle with curved sides to maintain an equal distance from any vertex to its opposing periphery. In 1876, Franz Reuleaux introduced constant breadth geometry other than circles in his book. In his book, a qualitative study on the kinematics of the Reuleaux triangle is presented and trajectories were presented in the book with a different reference frame. In the book, Franz Reuleaux demonstrated that when a Reuleaux triangle rotates inside a fixed square [24] the triangle covers $98.8 \%$ of the square. In 1917, James Watts invented the squarehole drill based on the kinematic properties of Reuleaux triangle [25]. This application was later studied by Figliolini [26] who presented the analytical expressions describing the motion vertex of the Reuleaux triangle when the triangle rotates inside its conjugate square. Another major industrial application of the Reuleaux triangle is the Wankel internal combustion engine. The Reuleaux triangle separates an epitrochoidal confinement into three chambers which changes volume while the Reuleaux triangle rotates. 


\subsubsection{Kinematic Analysis}

The kinematic analysis of the Reuleaux triangle cam is presented. Figure 3.1 shows the schematic diagram of the Reuleuax triangle driving mechanism of the Reuleaux triangle and its conjugate square.

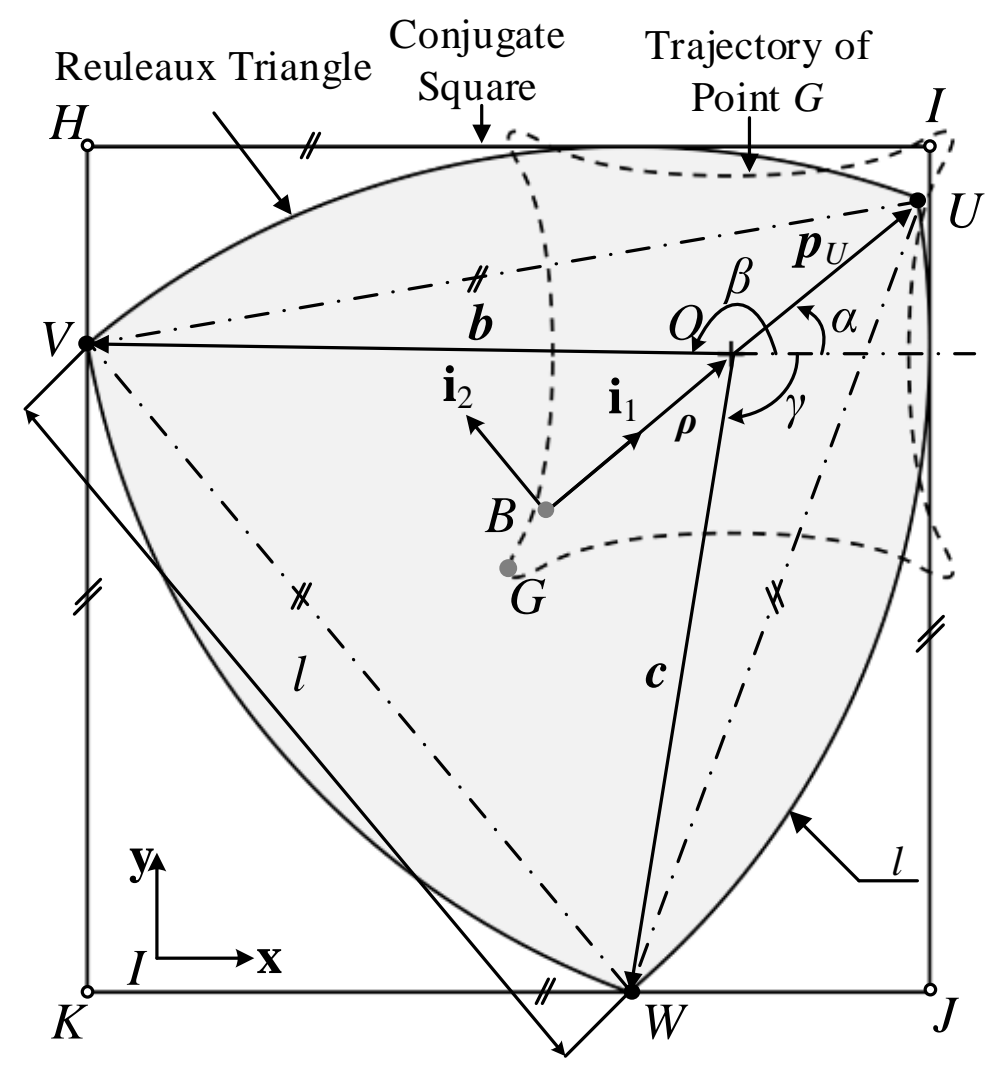

Figure 3.2: Schematic of Reuleaux triangle and its conjugate square

The triangle is composed of three vertices $U, V$, and $W$ with its centroid at $B$. The sides of the triangle are composed of three curves with its center at the opposite vertex. The conjugate square $H, I, J, K$ has side length $l$ [24]. Its centroid is represented as $G$. The triangle is set to rotate about a fixed point $O$ along vector $B U$ with a distance $\rho$ from $B$ with an input angle $\alpha$. The square than translates with respect to the rotation of the Reuleaux triangle. A body-attached frame of reference $\left(B, i_{1}, i_{2}\right)$ is attached to Reuleaux triangle at $B$ and $i_{1}$ is along $B U$. The Reuleaux triangle and its 
conjugate square form a single DOF system where rotation of the triangle results in planar displacement of the conjugate square on the inertial frame $(I, x, y)$.

The notation $\boldsymbol{p}_{j}^{(i)}$ represents the position of point $j$ in frame $I$. The scalar $x$ - and $y$-components of an arbitrary vector $Z$ will be represented as $Z x$ and $Z y$, respectively.

Notice that while the Reuleaux triangle rotates inside the conjugate square, at least two vertices are in contact with the sides of the conjugate square. Figure 3.1 show the case for $\pi / 6<\alpha<\pi / 3$. In this case, $V$ and $W$ are in contact with the conjugate square. During a full revolution, there are twelve states with a $\pi / 6$ interval. The vertices contacting the square are presented in Table 3.1 .

Table 3.1: States of Reuleaux Triangle Rotation

\begin{tabular}{|c|c|c|c|c|}
\hline Angle range & Vertices on $I J$ & Vertices on $K J$ & Vertices on $H K$ & Vertices on $\mathrm{HI}$ \\
\hline $0-\pi / 6$ & $\mathbf{U}$ & $\mathbf{W}$ & N/A & N/A \\
\hline$\pi / 6-\pi / 3$ & N/A & $\mathbf{W}$ & $\mathbf{V}$ & N/A \\
\hline$\pi / 3-\pi / 2$ & N/A & N/A & $\mathbf{V}$ & $\mathbf{U}$ \\
\hline$\pi / 2-2 \pi / 3$ & $\mathbf{W}$ & N/A & N/A & $\mathbf{U}$ \\
\hline $2 \pi / 3-5 \pi / 6$ & $\mathbf{W}$ & $\mathbf{V}$ & N/A & N/A \\
\hline $5 \pi / 6-\pi$ & N/A & $\mathbf{V}$ & $\mathbf{U}$ & N/A \\
\hline$\pi-7 \pi / 6$ & N/A & N/A & $\mathbf{U}$ & $\mathbf{W}$ \\
\hline $7 \pi / 6-4 \pi / 3$ & $\mathbf{V}$ & N/A & N/A & $\mathbf{W}$ \\
\hline $4 \pi / 3-3 \pi / 2$ & $\mathbf{V}$ & $\mathbf{U}$ & N/A & N/A \\
\hline $3 \pi / 2-5 \pi / 3$ & N/A & $\mathbf{U}$ & $\mathbf{W}$ & N/A \\
\hline $5 \pi / 3-11 \pi / 6$ & N/A & N/A & $\mathbf{W}$ & $\mathbf{V}$ \\
\hline $11 \pi / 6-2 \pi$ & $\mathbf{U}$ & N/A & N/A & $\mathbf{V}$ \\
\hline
\end{tabular}


The position of the conjugate square is given by its centroid location $\boldsymbol{p}_{G}^{(I)}$ which is given in Equation (1). Equation (1) is used to calculate the $G x$ and $G y$ component of the position vector $\boldsymbol{p}_{G}^{(I)}$ in the global reference frame based on Table 3.1.

$$
\mathbf{p}_{G}^{(I)}=\left\{\begin{array}{l}
\left(U x-\frac{l}{2}\right) \mathbf{x}+\left(W y+\frac{l}{2}\right) \mathbf{y}, \alpha=\left[0, \frac{\pi}{6}\right) \\
\left(V x+\frac{l}{2}\right) \mathbf{x}+\left(W y+\frac{l}{2}\right) \mathbf{y}, \alpha=\left[\frac{\pi}{6}, \frac{\pi}{3}\right) \\
\left(V x+\frac{l}{2}\right) \mathbf{x}+\left(U y-\frac{l}{2}\right) \mathbf{y}, \alpha=\left[\frac{\pi}{3}, \frac{\pi}{2}\right) \\
\left(W x-\frac{l}{2}\right) \mathbf{x}+\left(U y-\frac{l}{2}\right) \mathbf{y}, \alpha=\left[\frac{\pi}{2}, \frac{2 \pi}{3}\right) \\
\left(W x-\frac{l}{2}\right) \mathbf{x}+\left(V y+\frac{l}{2}\right) \mathbf{y}, \alpha=\left[\frac{2 \pi}{3}, \frac{5 \pi}{6}\right) \\
\left(U x+\frac{l}{2}\right) \mathbf{x}+\left(W y-\frac{l}{2}\right) \mathbf{y}, \alpha=\left[\pi, \frac{7 \pi}{6}\right) \\
\left(V x-\frac{l}{2}\right) \mathbf{x}+\left(W y-\frac{l}{2}\right) \mathbf{y}, \alpha=\left[\frac{7 \pi}{6}, \frac{4 \pi}{3}\right) \\
\left(V x-\frac{l}{2}\right) \mathbf{x}+\left(U y+\frac{l}{2}\right) \mathbf{y}, \alpha=\left[\frac{5 \pi}{3}, \frac{3 \pi}{2}\right) \\
\left(W x+\frac{l}{2}\right) \mathbf{x}+\left(U y+\frac{l}{2}\right) \mathbf{y}, \alpha=\left[\frac{3 \pi}{2}, \frac{5 \pi}{3}\right) \\
\left(W x+\frac{l}{2}\right) \mathbf{x}+\left(V y+\frac{l}{2}\right) \mathbf{y}, \alpha=\left[\frac{5 \pi}{3}, \frac{11 \pi}{6}\right) \\
\left(U x-\frac{l}{2}\right) \mathbf{x}+\left(V y+\frac{l}{2}\right) \mathbf{y}, \alpha=\left[\frac{11 \pi}{6}, \pi\right)
\end{array}\right.
$$

However, the vertex positions of the Reuleaux triangle defined as $\boldsymbol{p}_{U}^{(I)}, \boldsymbol{p}_{V}^{(I)}$, and $\boldsymbol{p}_{W}^{(I)}$ in Equation (1) can be computed with a coordinate transformation from $B$ to $I$ using Equation (2):

$$
\begin{aligned}
& \mathbf{p}_{U}^{(I)}=\mathbf{T}_{B}^{I}(\alpha)\left(-\mathbf{\rho}^{(B)}+\mathbf{p}_{U}^{(B)}\right)+\mathbf{p}_{O}^{(I)} \\
& \mathbf{p}_{V}^{(I)}=\mathbf{T}_{B}^{I}(\alpha)\left(-\mathbf{\rho}^{(B)}+\mathbf{p}_{V}^{(B)}\right)+\mathbf{p}_{O}^{(I)} \\
& \mathbf{p}_{W}^{(I)}=\mathbf{T}_{B}^{I}(\alpha)\left(-\mathbf{\rho}^{(B)}+\mathbf{p}_{W}^{(B)}\right)+\mathbf{p}_{O}^{(I)}
\end{aligned}
$$

Here, $\boldsymbol{T}_{B}^{I}(\alpha)$ represents the rotation matrix from frame $B$ to $I$ for an input angle of $\alpha$. The vertices and rotational offsets in the body fixed frame $B$ are defined in Equation (3). 


$$
\begin{aligned}
& \mathbf{p}_{U}^{(B)}=\frac{l}{\sqrt{3}} \mathbf{i}_{l}, \mathbf{p}_{V}^{(B)}=-\frac{l}{2 \sqrt{3}} \mathbf{i}_{1}+\frac{l}{2} \mathbf{i}_{2} \\
& \mathbf{p}_{W}^{(B)}=-\frac{l}{2 \sqrt{3}} \mathbf{i}_{1}-\frac{l}{2} \mathbf{i}_{2}, \boldsymbol{\rho}^{(B)}=\rho \mathbf{i}_{1}
\end{aligned}
$$

Substituting Equation (3) into Equation (1) yields the coordinate of $G$ as a function of the offset $\rho$, as described in Equation (4):

$$
\begin{aligned}
& {\left[\frac{l}{2}\left(\frac{2}{\sqrt{3}}-\frac{\rho}{l}\right) \cos (\alpha)-\frac{l}{2}\right] \mathbf{x}+\left[\frac{l}{2}+\frac{l}{2}\left(-\cos (\alpha)-\left(\frac{1}{\sqrt{3}}+\frac{\rho}{l}\right) \sin (\alpha)\right)\right] \mathbf{y}, \alpha=\left[0, \frac{\pi}{6}\right)} \\
& {\left[\frac{l}{2}+\frac{l}{2}\left(-\left(\frac{1}{\sqrt{3}}+\frac{\rho}{l}\right) \cos (\alpha)-\sin (\alpha)\right)\right] \mathbf{x}+\left[\frac{l}{2}+\frac{l}{2}\left(-\cos (\alpha)-\left(\frac{1}{\sqrt{3}}+\frac{\rho}{l}\right) \sin (\alpha)\right)\right] \mathbf{y}, \alpha=\left[\frac{\pi}{6}, \frac{\pi}{3}\right)} \\
& {\left[\frac{l}{2}+\frac{l}{2}\left(-\left(\frac{1}{\sqrt{3}}+\frac{\rho}{l}\right) \cos (\alpha)-\sin (\alpha)\right)\right] \mathbf{x}+\left[\frac{l}{2}\left(\frac{2}{\sqrt{3}}-\frac{\rho}{l}\right) \sin (\alpha)-\frac{l}{2}\right] \mathbf{y}, \alpha=\left[\frac{\pi}{3}, \frac{\pi}{2}\right)} \\
& {\left[\frac{l}{2}\left(-\left(\frac{1}{\sqrt{3}}+\frac{\rho}{l}\right) \cos (\alpha)+\sin (\alpha)\right)-\frac{l}{2}\right] \mathbf{x}+\left[\frac{l}{2}\left(\frac{2}{\sqrt{3}}-\frac{\rho}{l}\right) \sin (\alpha)-\frac{l}{2}\right] \mathbf{y}, \alpha=\left[\frac{\pi}{2}, \frac{2 \pi}{3}\right)} \\
& {\left[\frac{l}{2}\left(-\left(\frac{1}{\sqrt{3}}+\frac{\rho}{l}\right) \cos (\alpha)+\sin (\alpha)\right)-\frac{l}{2}\right] \mathbf{x}+\left[\frac{l}{2}+\frac{l}{2}\left(\cos (\alpha)-\left(\frac{1}{\sqrt{3}}+\frac{\rho}{l}\right) \sin (\alpha)\right)\right] \mathbf{y}, \alpha=\left[\frac{2 \pi}{3}, \frac{5 \pi}{6}\right)} \\
& {\left[\frac{l}{2}\left(\frac{2}{\sqrt{3}}-\frac{\rho}{l}\right) \cos (\alpha)+\frac{l}{2}\right] \mathbf{x}+\left[\frac{l}{2}+\frac{l}{2}\left(\cos (\alpha)-\left(\frac{1}{\sqrt{3}}+\frac{\rho}{l}\right) \sin (\alpha)\right)\right] \mathbf{y}, \alpha=\left[\frac{5 \pi}{6}, \pi\right)} \\
& {\left[\frac{l}{2}\left(\frac{2}{\sqrt{3}}-\frac{\rho}{l}\right) \cos (\alpha)+\frac{l}{2}\right] \mathbf{x}+\left[\frac{l}{2}\left(-\cos (\alpha)-\left(\frac{1}{\sqrt{3}}+\frac{\rho}{l}\right) \sin (\alpha)\right)-\frac{l}{2}\right] \mathbf{y}, \alpha=\left[\pi, \frac{7 \pi}{6}\right)} \\
& {\left[\frac{l}{2}\left(-\left(\frac{1}{\sqrt{3}}+\frac{\rho}{l}\right) \cos (\alpha)-\sin (\alpha)\right)-\frac{l}{2}\right] \mathbf{x}+\left[\frac{l}{2}\left(-\cos (\alpha)-\left(\frac{1}{\sqrt{3}}+\frac{\rho}{l}\right) \sin (\alpha)\right)-\frac{l}{2}\right] \mathbf{y}, \alpha=\left[\frac{7 \pi}{6}, \frac{4 \pi}{3}\right)} \\
& {\left[\frac{l}{2}\left(-\left(\frac{1}{\sqrt{3}}+\frac{\rho}{l}\right) \cos (\alpha)-\sin (\alpha)\right)-\frac{l}{2}\right] \mathbf{x}+\left[\frac{l}{2}\left(\frac{2}{\sqrt{3}}-\frac{\rho}{l}\right) \sin (\alpha)+\frac{l}{2}\right] \mathbf{y}, \alpha=\left[\frac{4 \pi}{3}, \frac{3 \pi}{2}\right)} \\
& {\left[\frac{l}{2}+\frac{l}{2}\left(-\left(\frac{1}{\sqrt{3}}+\frac{\rho}{l}\right) \cos (\alpha)+\sin (\alpha)\right)\right] \mathbf{x}+\left[\frac{l}{2}\left(\frac{2}{\sqrt{3}}-\frac{\rho}{l}\right) \sin (\alpha)+\frac{l}{2}\right] \mathbf{y}, \alpha=\left[\frac{3 \pi}{2}, \frac{5 \pi}{3}\right)} \\
& {\left[\frac{l}{2}+\frac{l}{2}\left(-\left(\frac{1}{\sqrt{3}}+\frac{\rho}{l}\right) \cos (\alpha)+\sin (\alpha)\right)\right] \mathbf{x}+\left[\frac{l}{2}\left(\cos (\alpha)-\left(\frac{1}{\sqrt{3}}+\frac{\rho}{l}\right) \sin (\alpha)\right)-\frac{l}{2}\right] \mathbf{y}, \alpha=\left[\frac{5 \pi}{3}, \frac{11 \pi}{6}\right)} \\
& {\left[\frac{l}{2}\left(\frac{2}{\sqrt{3}}-\frac{\rho}{l}\right) \cos (\alpha)-\frac{l}{2}\right] \mathbf{x}+\left[\frac{l}{2}\left(\cos (\alpha)-\left(\frac{1}{\sqrt{3}}+\frac{\rho}{l}\right) \sin (\alpha)\right)-\frac{l}{2}\right] \mathbf{y}, \alpha=\left[\frac{11 \pi}{6}, \pi\right)}
\end{aligned}
$$

\subsubsection{Conjugate Square Trajectory}

In Equation (4), the trajectory of point $\mathrm{G}$ denoted as $\pi_{\mathrm{i}}$ for various offset values $\rho$, are illustrated in Figure 3.2. The profiles are normalized with respect to the triangle length, $l$, where $\alpha=[0,2 \pi]$. Note that the trajectory profile varies with respect to the different offsets of $\rho$. The trajectories $P_{1}$, $P_{2}, P_{3}, P_{4}$, and $P_{8}$ are shown in Figure 3.2(a) and the trajectories $P_{5}, P_{6}$, and $P_{7}$ are shown in Figure 
3.2(b) at a scale of 3:1. The following information from reference [24] is reworded slightly for clarification below:
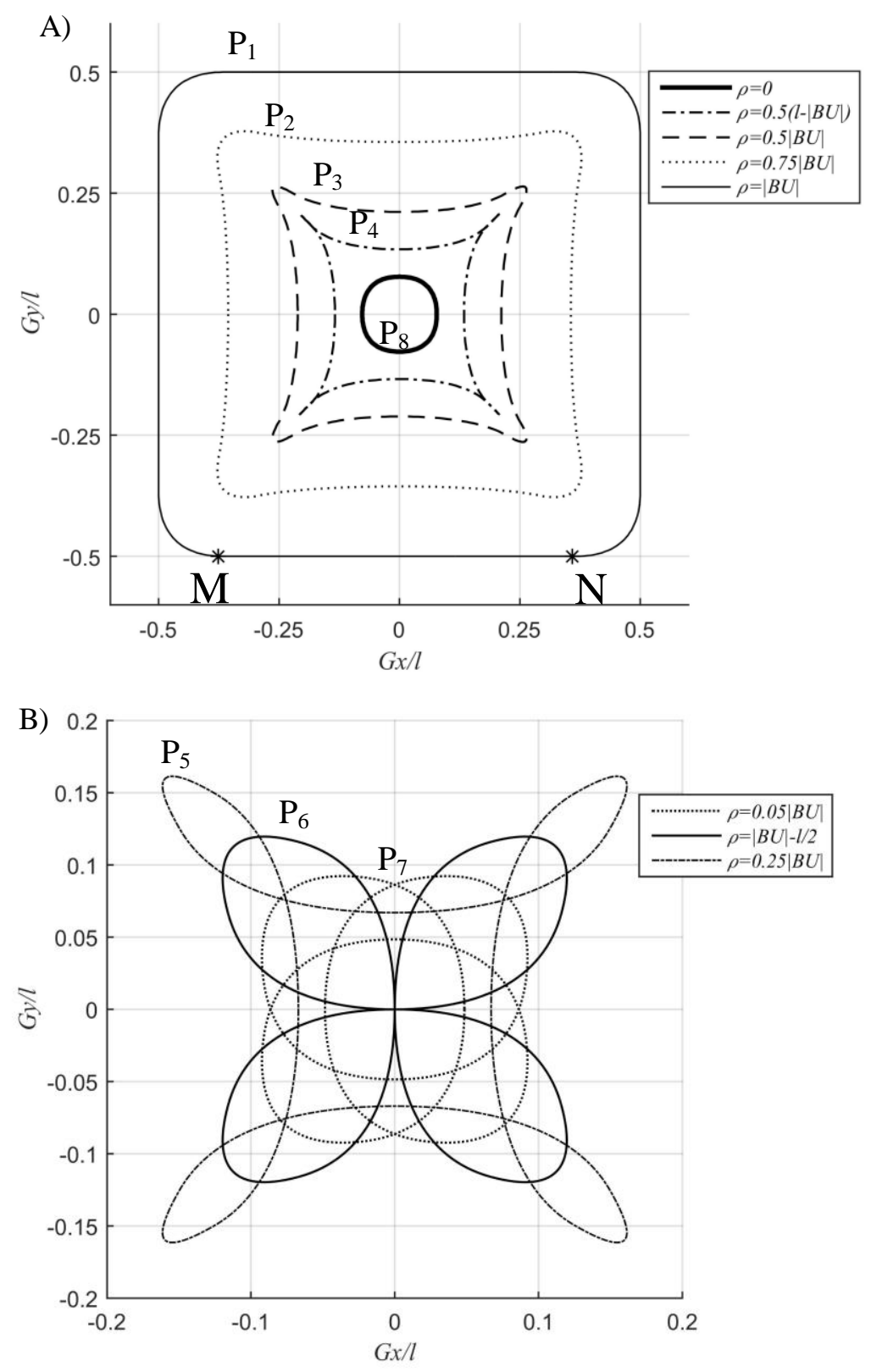

Figure 3.3: Trajectory profiles of point $G$ normalized for various offset values $\rho$

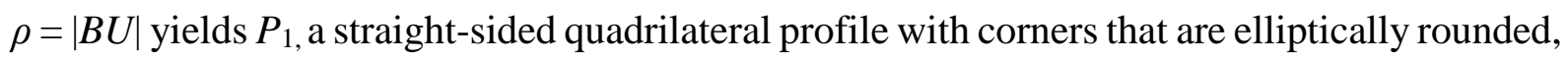
shown in Figure 4(A). For values of $\rho$ satisfying the inequality $0.5(l-|B U|)<\rho<|B U|$, the trajectories $P_{2}$ and $P_{3}$ are obtained, and represent a concaved sided quadrilateral with round corners. 
For $\rho=0.5(l-|B U|)$ the trajectory denoted as $P_{4}$ represents a superellipse, or Lamé curve which composed of four elliptical curves that are tangent to its neighboring curve.

For values of $\rho$ satisfying the inequality $(|B U|-l / 2)<\rho<0.5(l-|B U|)$, the trajectory $P_{5}$ resembles a profile with four concave elliptical curves that intersect their neighboring curves to form loops.

Similarly, for $\rho=(|B U|-l / 2)$, the $\mathrm{P}_{6}$ trajectory loop curves are tangent to one another, forming what is referred to as a homocentral form of $P_{5}$. For $0<\rho<(|B U|-l / 2)$ the $\mathrm{P}_{7}$ trajectory forms four elliptical curves that intersect with each other. For $\rho=0$, the $P_{8}$ trajectory forms a profile with four convex elliptical curves that are tangent to neighboring curves [24].

\subsubsection{Generalized Reuleaux triangle}

In the designing of the Reuleaux triangle cam, a generalized Reuleaux triangle is used to prevent the wear and concentration of stress at the tip. As shown in Figure 3.3, a generalized Reuleaux triangle is achieved by setting the radius of the curve larger than the side length of the equilateral triangle, and each side is tangentially connected by round curves with its center located at the nearest triangle vertex. Hence, a generalized Reuleaux triangle is also a contact breadth geometry which can rotate inside a square. In Figure 3.3, a generalized Reuleaux triangle $U V W$ and its conjugate square $H^{\prime} I^{\prime} J^{\prime} K$ ' are represented by the solid line. The Reuleaux triangle $U V W$, which the generalized Reuleaux triangle generated is from, and its conjugate square HIJK are represented by a dashed line.

Using the same configurations in Section 2, the generalized Reuleaux triangle rotates about point $O$ with an offset $\rho$ from its centroid $B$. The contact point of the generalized Reuleaux triangle can be chosen such that it is on the curve with a radius of $d$. 


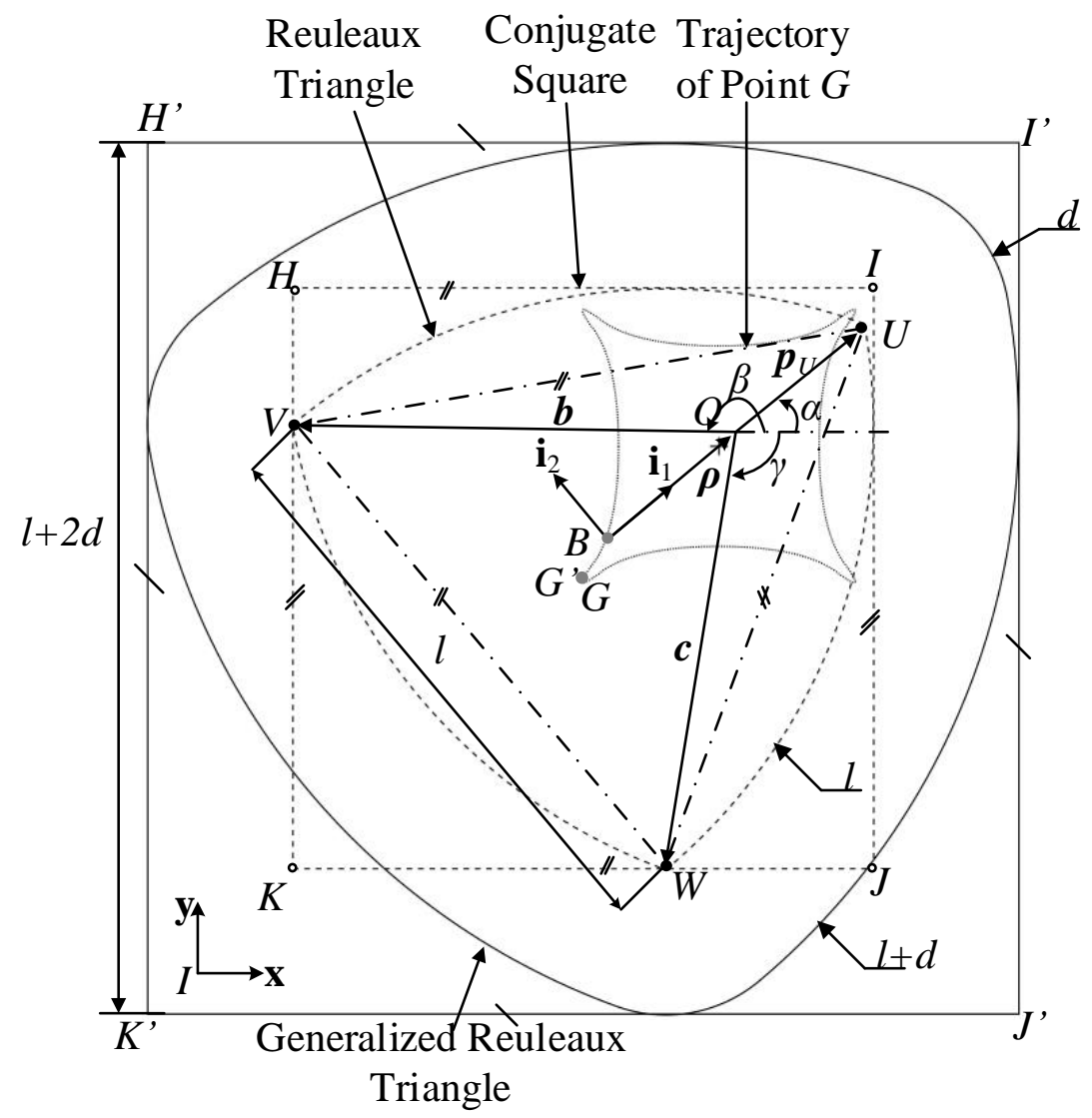

Figure 3.4: Generalized Reuleaux triangle

The contact point of the generalized Reuleaux triangle $U V W$ with respect to the conjugate square can be computed with the vertices of the Reuleaux triangle $U V W$ that is in contact with the conjugate square. $C_{1}{ }^{\prime}$ is defined as the contact point of the generalized Reuleaux triangle and the conjugate square $H^{\prime} I^{\prime} J J^{\prime} K^{\prime}$ on the line $H^{\prime} K^{\prime}$ or $I^{\prime} J, C_{2}$ ' is defined as the contact point on the line $H^{\prime} I^{\prime}$ and $K^{\prime} J^{\prime} . C_{l}$ is defined as the contact point of the Reuleaux triangle and the conjugate square $H I J K$ on the line $H K$ or $I J$ while $C_{2}$ is defined as the contact point on the line $H I$ and $K J$. Therefore, the $x$ component of $C_{1}$ can be computed as

$$
C_{1}^{\prime} x=C_{1} x+\operatorname{sign}\left(C_{1}\right) d
$$

and $y$ component can be computed as 


$$
C_{2}^{\prime} y=C_{2} y+\operatorname{sign}\left(C_{2}\right) d
$$

The sign of $d$ is determined by the location of the contact point, which is the opposite as the sign of each of the $l / 2$ terms in Equation (1). It should be noted that in Figure 3.3, the side length of the conjugate square is $l+2 d$, therefore, the position vector of the conjugate square $H^{\prime} I^{\prime} J^{\prime} K^{\prime}$ 'can be derived as

$$
\mathbf{p}_{G^{\prime}}^{(I)}=\left(C_{1}^{\prime} x-\operatorname{sign}\left(C_{1}^{\prime} x\right) \frac{l+2 d}{2}\right) \mathbf{x}+\left(C_{2}^{\prime} y-\operatorname{sign}\left(C_{2}^{\prime} y\right) \frac{l+2 d}{2}\right) \mathbf{y}
$$

Note that the sign of Equation (5) and (6) is opposite with respect to Equation (1), therefore, the $\mathrm{d}$ term is canceled out during the calculation which yields the same results as Equation (4). Therefore, Equation (4) is also the trajectory function of the generalized Reuleaux triangle and the trajectory profile remains the same.

\subsubsection{Friction Between the Cam and the Cam-follower}

With reference to Figure 3.4, the Reuleaux triangle is rotating about a fixed point in the space. Here $C_{3}$ ' and $C_{4}$ ' are defined as the point of the generalized Reuleaux triangle that are in contact with the conjugate square. The position vectors of $C_{3}$ ' and $C_{4}$ ' are calculated as

$$
\begin{aligned}
& \mathbf{O C}_{3}^{\prime}=\mathbf{O C}_{1}^{\prime}-\operatorname{sign}\left(C_{1}^{\prime} x\right)(l+2 d) \mathbf{x} \\
& \mathbf{O C}_{4}^{\prime}=\mathbf{O C}_{2}^{\prime}-\operatorname{sign}\left(C_{2}^{\prime} y\right)(l+2 d) \mathbf{y}
\end{aligned}
$$

The velocity of the contact points on the Reuleaux triangle can be derived as

$$
v_{i}^{C}=\dot{\boldsymbol{\alpha}} \times \mathbf{O C}_{i}^{\prime}
$$

Here, superscript $C$ means the contact point on the Reuleaux triangle cam, subscript i means the $i$-th contact point.

The velocity of the cam follower can be derived as 


$$
v_{f}=\frac{d}{d t} \mathbf{p}_{G}^{I}
$$

The relative velocity of the contact points on the Reuleaux triangle cam and the cam follower can be computed as

$$
\mathbf{v}_{r}=\mathbf{v}_{i}^{C}-\mathbf{v}_{f}
$$

Here, the angular velocity of the cam is set to be $1 \mathrm{rad} / \mathrm{s}$. In a full revolution, the relative velocity of the contact point in the horizontal and vertical directions are shown in Figure 3.5.
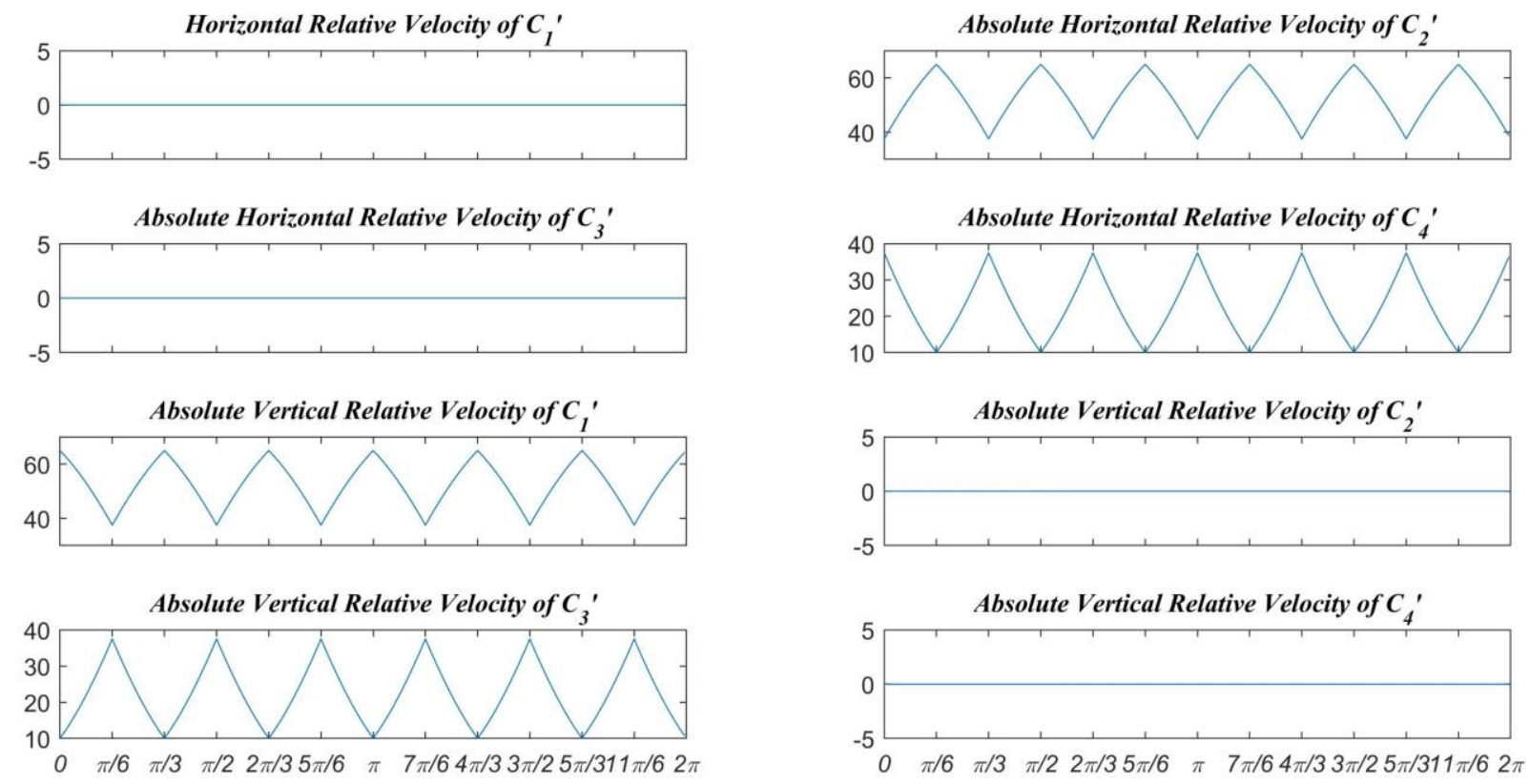

Figure 3.5: Relative velocity of the contact points

With reference to Figure 3.5, the absolute values of relative velocity are used to analyze the friction mode. For $C_{1}$ ' and $C_{3}$ ', the horizontal relative velocity shows zero, $C_{2}$ ' and $C_{4}$ 'shown zero vertical relative velocity during a full revolution. Hence, there is no sliding motion along these movements. However, for $C_{1}$ ' and $C_{3}$ ', the vertical relative velocities show non-zero and $C_{2}$ ' and $C_{4}$ ' horizontal velocities show non-zero. Therefore, sliding motion can be detected between the 
Reuelaux triangle cam and the cam follower. The friction between the cam and the cam follower can be described as a combination of sliding friction and rolling friction [27].

\subsection{Cam - follower mechanism design}

\subsubsection{Cam Design}

This section presents the mechanism design of the cam-follower mechanism. In Chapter 2, a straight line foot trajectory is desired. Therefore, the cam is designed based on the generalized Reuleaux triangle, the offset of the rotation axis is $\rho=|B U|=(\sqrt{3} / 3) l$. Here, $l$ is defined as in section 3.1

The cam assembly is formed by three generalized Reuleaux. Three generalized Reuleaux triangle are arranged in a sandwich form. The outer layers are slightly larger than the inner layer of the cam assembly. The inner layer coupled with the foot conjugate square forms the camfollower mechanism. The two outer layer restricts the axial motion of the Reuleaux triangle to prevent the dismantling of the mechanism.

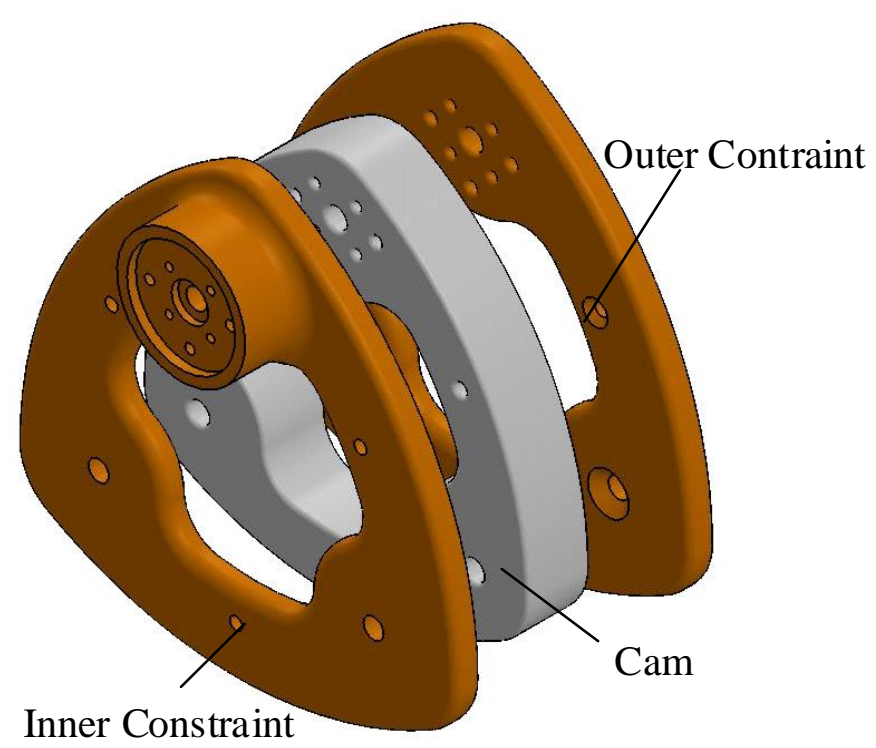

Figure 3.6: CAD model of the cam assembly 
The robot is designed with a step stroke and step height of $75 \mathrm{~mm}$. Therefore, for the inner layer of the cam assembly, the side length $l$ of the equilateral triangle from which the generalized Reuleaux triangle generated is set to be $75 \mathrm{~mm}$. To allow the installation of the actuator and to avoid the stress concentration at the tip of the cam, $d$ is set to be $15 \mathrm{~mm}$. The inner constraint connects to the motor and transmits torque from the motor to the cam. Note that in Figure 3.6, two Reuleaux triangles were used to determine the position of the constraint and mounting holes. The smaller Reuleaux triangle determines the mounting holes while the larger Reuleaux triangle generated the profile of the constraint. A cylinder profile extruded from the generalized Reuleaux triangle profile was connected to the motor with seven pins. The outer constraint shares the same layout as the inner constraint. However, the cylindrical motor connector is removed.

\subsubsection{Foot assembly}

Figure 3.7 shows the foot assembly of the robot. The foot assembly consists of a conjugate square cam follower (box) and the box and the foot was connected by screws. The box follows the cam to create desired foot trajectory while the foot provides support such that the robot could walk without fell.

The corner of the square hole is rounded to avoid stress concentration. The side length of the square is $0.30 \mathrm{~mm}$ longer than the theoretical conjugate square which allows for tolerance between the cam and the follower. The two upper holes connected to the double four-bar mechanism and the lower holes connected to the foot. 


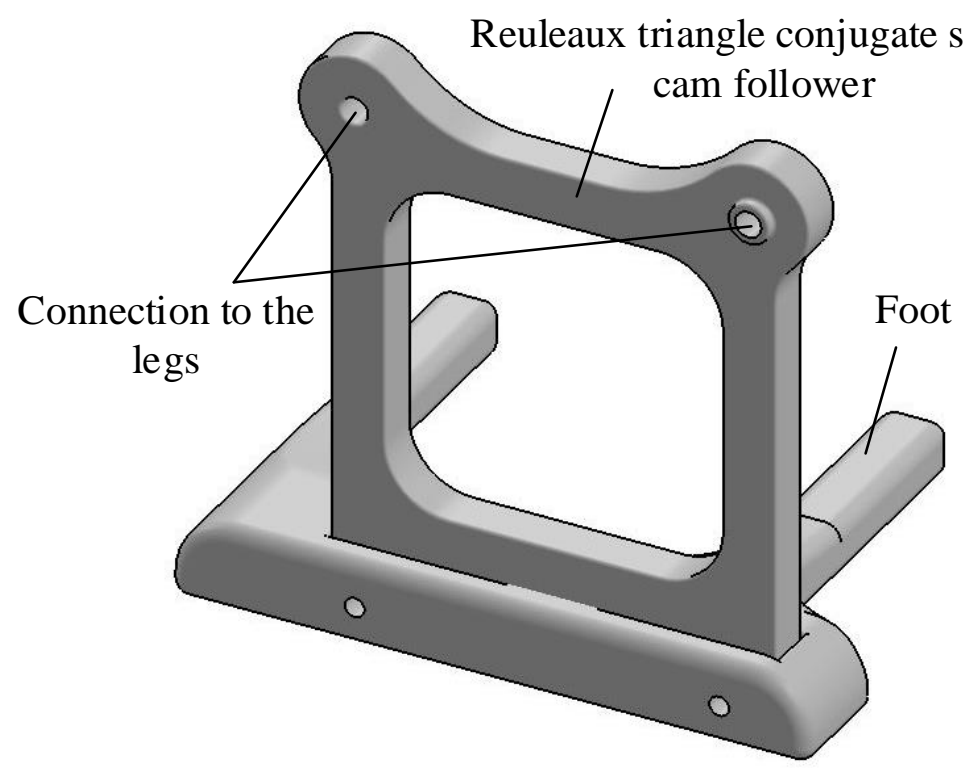

Figure 3.7: CAD model of the foot assembly

The foot is designed such that it can maintain a standing position while one foot is lifted from the ground. Therefore, the "toes" of the foot is longer than half of the total width of the robot. The position of ties was offset from the center to avoid the interference during turning, which utilizes a differential drive method.

\subsection{Robot Body}

The foot assembly and the cam assembly is connected to the body via a double four-bar mechanism and the motor, respectively. The double four-bar mechanism resembles the leg of the robot and restricts the rotational motion of the foot such that the foot maintains a constant orientation during walking gaits. The motor actuates the cam which drives the robot forward. The diagram of the body is shown in Figure 3.8. The body contains two motors, motor mounts, and the four-bar mechanism connector. 


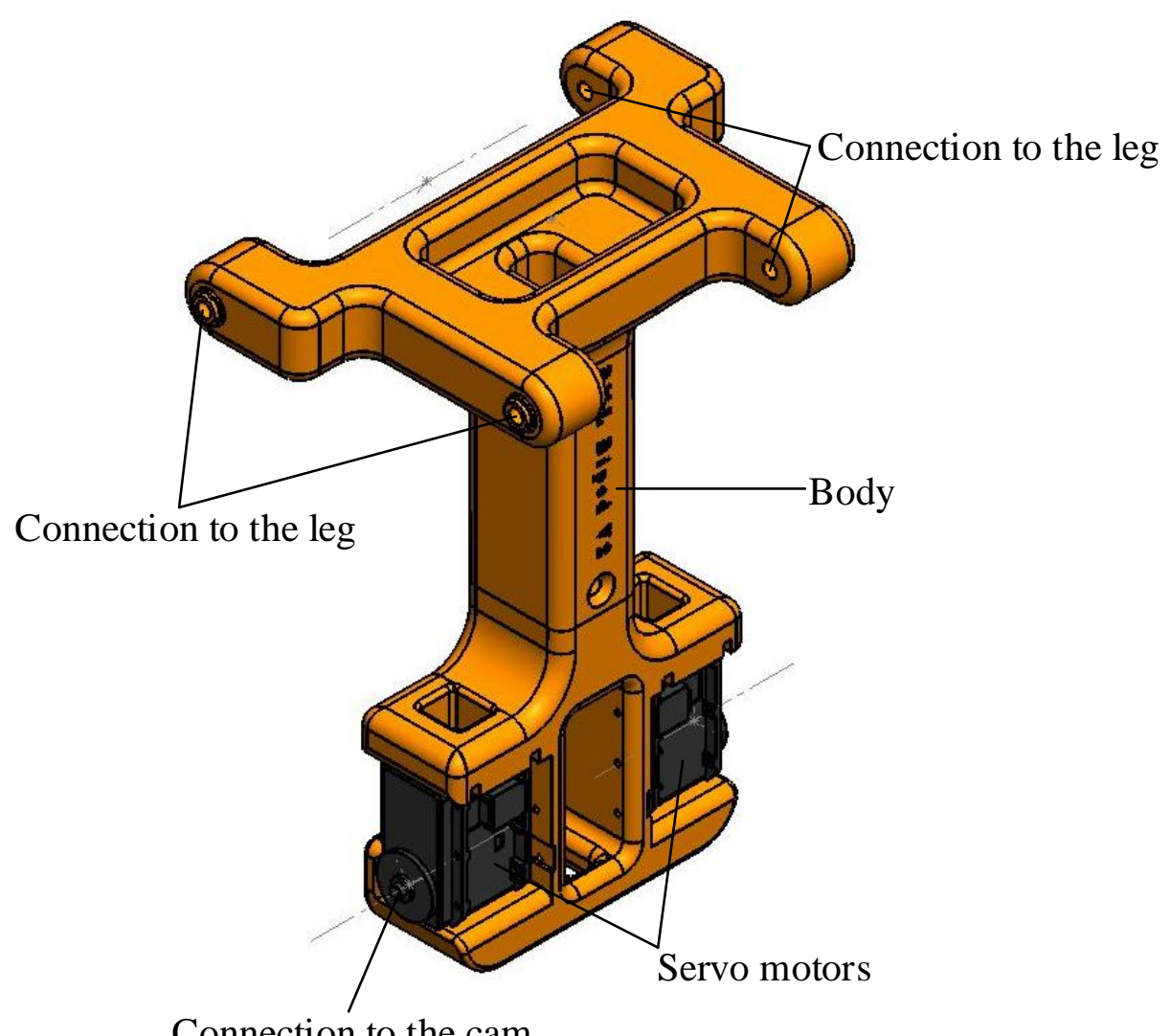

Connection to the cam

Figure 3.8: CAD model of the body assembly

Note that in Figure 3.8, the connector that connects to the four-bar mechanism is designed with a unique shape rather than a rectangle. These feature results from the arrangement of the links of the four-bar mechanism. The links in the four-bar mechanism are staggered such that the torque caused by constraint force at each joint cancels out. The motor is mounted at the bottom of the body. The motor directly connects to the generalized Reuleaux triangle cam through a set of pins. 


\section{CHAPTER 4}

\section{TRAJECTORY PLANNING}

The purpose of the trajectory planning is to achieve two goals: (1) finding the desired conjugate square profile such that the robot could maintain a constant body height during a walking gait and (2) finding a desired Reuleaux triangle angle-time relation such that the robot could walk with constant velocity.

\subsection{Foot Trajectory}

A walking gait consists of support phase and a swing phase. The support phase is defined as the foot trajectory portion that is contacting the ground, while the swing phase is defined as the foot swings in mid-air from the rear of the robot to the front of the robot. In a walking gait, as discussed in Chapter 3, it is desirable that the robot maintains a constant body height, which minimizes the energy consumptions and reduces the instability induced by the fluctuation. A straight line support phase of the foot trajectory should be achieved. The RML-V2 is a single DOF mechanism with fixed foot (conjugate square) trajectory that only varies with respect to $\rho$, thus, the process of foot trajectory planning involves: (1) selecting $\rho$ such that the foot trajectory is optimal for walking, and (2) synthesizing mechanism design parameters to obtain a desirable step length and step height.

In Figure 4.2(A), trajectory profile $P_{1}$ presents a straight-sided quadrilateral with elliptically curved corners. Therefore, the straight side of the profile $P_{1}$ can be used as the foot trajectory. Therefore, the rotational offset distance from the Reuleaux triangle centroid should be set to $\rho=|B U|=l / \sqrt{3}$ to generate this foot trajectory. Figure 5.1 shows the trajectory profile with 
respect to input angle $\alpha$. In Figure 4.1, the support phase is defined as the solid line MN and the support phase is the dashed profile.

Step length is the length of the support phase of the trajectory, in this case, the step length is defined as the length of solid line MN. Note that in Figure 4.1, the step length is not the stroke of the foot trajectory. Therefore, we define step stroke to be the horizontal distance the foot travels during a gait cycle, and step height is the vertical distance the foot travels in a gait cycle.

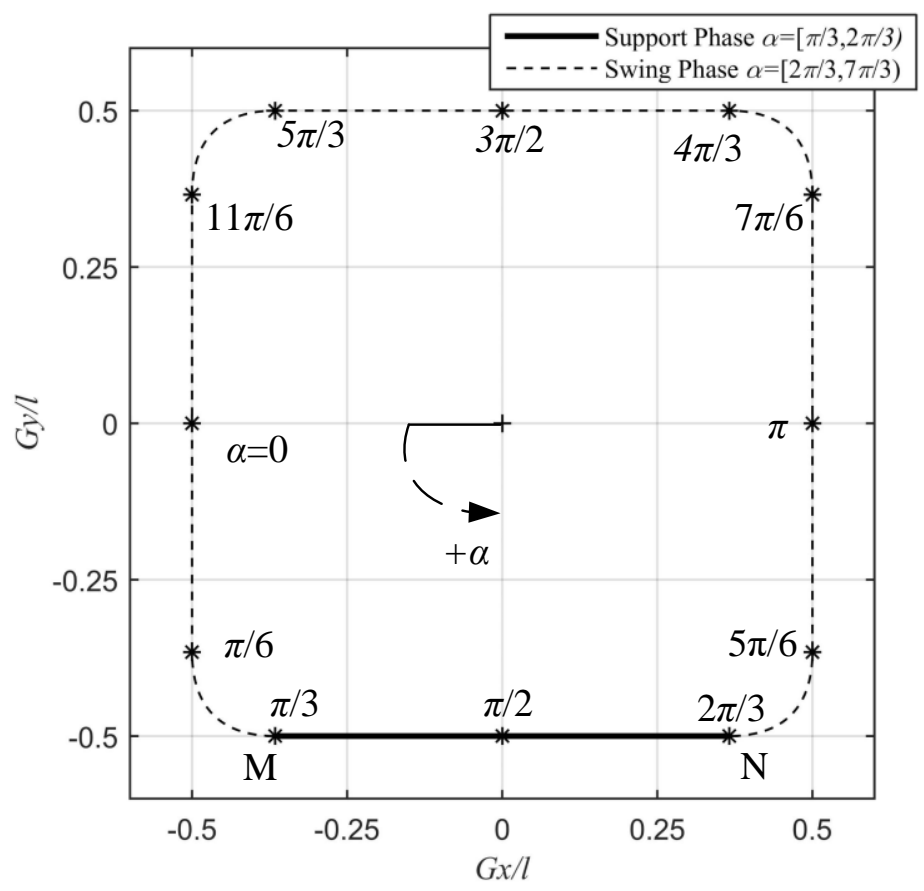

Figure 4.1: Foot trajectory profile with input angle $\alpha$

As seen in Figure 4.1, the support phase of the robot initiates when $\alpha=\pi / 3$ and terminates when $\alpha=2 \pi / 3$. Substituting these values into Equation (4), the position vector OM and ON are calculated as

$$
\begin{aligned}
& \mathbf{O M}=\left(-l \frac{\sqrt{3}-1}{2},-\frac{l}{2}\right)^{T} \\
& \mathbf{O N}=\left(-l \frac{\sqrt{3}-1}{2},-\frac{l}{2}\right)^{T}
\end{aligned}
$$


$O M$ and $O N$ are the position vector of $O G$ when $G$ collide with $M$ and $N$ respectively.

Hence, the step length is the norm of vector $M N$, which yields

$$
M N=|\mathbf{O N}-\mathbf{O M}|=l(\sqrt{3}-1)
$$

Therefore, the step length is equivalent to $l(\sqrt{3}-1)$.

With reference to Equation (4) and Figure 4.1, the profile shows a straight-sided quadrilateral and the straight line profiles achieved when $\alpha \in(n \pi / 2-\pi / 6, n \pi / 2+\pi / 6)$, where $n$ is an integer.

For $\alpha \in(0, \pi / 6]$ or $\alpha \in(11 \pi / 6,2 \pi]$, the trajectory profile is represented as a vertical straight line which can be described as $\mathrm{x}=-l / 2$. For $\alpha \in(\pi / 3,2 \pi / 3]$, the profile is a horizontal straight line which can be described as $\mathrm{y}=-l / 2$. For $\alpha \in(5 \pi / 6,7 \pi / 6]$, the profile can be described as $\mathrm{x}=l / 2$, indicating a straight vertical line and for $\alpha \in(4 \pi / 3,5 \pi / 3]$, the profile shows a horizontal straight line which can be described as $y=l / 2$. Therefore, the step stroke and step length can be calculated by finding the distance between two vertical lines and two horizontal lines respectively. The step stroke and step length are

$$
\begin{aligned}
& d_{\text {stroke }}=l \\
& h=l
\end{aligned}
$$

Where $d_{\text {stroke }}$ represents step stroke and $\mathrm{h}$ represents step height.

These relations can be used to synthesize the dimensions of the Reuleaux triangle in order to achieve a desirable step length and height.

\subsection{Gait Sequencing}

The previous section indicates that the optimal support phase only takes up $1 / 6$ of total input angle during a full revolution of the cam. Therefore, in order to achieve a constant body height 
during walking, the swing phase and the support phase should be sequenced such that one leg initiates the support phase while the other leg simultaneously initiates the swing phase and vice versa. Otherwise, if the legs are not sequenced, the robot body will fluctuate vertically and may cause instances of instability.

Gait cycle period is defined as the time $(T)$ required to complete a full cycle by the foot, which is the sum of the time of support phase and swing phase. During the support phase of one foot, the robot travels a distance equivalent to $M N$. The robot is designed such that it is walking with a constant velocity $v$. Given that the gait cycle is $T$, the forward walking velocity can be derived as

$$
v=2 \frac{M N}{T}=\frac{l}{T}(2 \sqrt{3}-2)
$$

The robot utilizes bipedal locomotion, and when one foot initiates support phase, the other foot initiates swing phase, hence, the time for swing phase and support phase should be equal. Thus, $t_{\text {support }}=t_{\text {swing }}=T / 2$.

Assuming a no-slip condition, and when one foot is in support phase while the other is in the swing phase, the position of the robot in each step can be written as

$$
\mathbf{p}_{\text {robot }}=f\left(\alpha_{l}, \alpha_{r}\right)=\left\{\begin{array}{l}
-\mathbf{p}_{G}^{(I)}\left(\alpha_{l}\right)+\mathbf{p}_{0}, \alpha_{l} \in\left[\frac{\pi}{3}, \frac{2 \pi}{3}\right) \& \alpha_{r} \notin\left[\frac{\pi}{3}, \frac{2 \pi}{3}\right) \\
-\mathbf{p}_{G}^{(I)}\left(\alpha_{r}\right)+\mathbf{p}_{0}, \alpha_{r} \in\left[\frac{\pi}{3}, \frac{2 \pi}{3}\right) \& \alpha_{l} \notin\left[\frac{\pi}{3}, \frac{2 \pi}{3}\right]
\end{array}\right.
$$

Here, $\boldsymbol{P}_{0}$ denotes the distance the robot traveled, and the value of $\boldsymbol{P}_{0}$ changes when each foot initiates its support phase. The value of $\boldsymbol{P}_{0}$ is calculated as

$$
\mathbf{p}_{0}=n l(\sqrt{3}-1) \mathbf{x}+\frac{l}{2} \mathbf{y}
$$

Where $n$ represents the number of steps performed. 
The velocity of the robot can then be acquired by taking the time derivative of $\boldsymbol{P}_{\text {robot. }}$ Therefore, the velocity of the robot is

$$
\mathbf{u}=\frac{d}{d t} \mathbf{p}_{\text {robot }}=\left\{\begin{array}{l}
\left(-\frac{d \alpha_{l}}{d t} \frac{l}{2} \cos \left(\alpha_{l}\right)+\frac{d \alpha_{l}}{d t} \frac{\sqrt{3}}{2} l \sin \left(\alpha_{l}\right)\right) \mathbf{x}, \alpha_{l} \in\left[\frac{\pi}{3}, \frac{\pi}{2}\right) \& \alpha_{r} \notin\left[\frac{\pi}{3}, \frac{2 \pi}{3}\right) \\
\left(\frac{d \alpha_{l}}{d t} \frac{l}{2} \cos \left(\alpha_{l}\right)+\frac{d \alpha_{l}}{d t} \frac{\sqrt{3}}{2} l \sin \left(\alpha_{l}\right)\right) \mathbf{x}, \alpha_{l} \in\left[\frac{\pi}{2}, \frac{2 \pi}{3}\right) \& \alpha_{r} \notin\left[\frac{\pi}{3}, \frac{2 \pi}{3}\right) \\
\left(-\frac{d \alpha_{r}}{d t} \frac{l}{2} \cos \left(\alpha_{r}\right)+\frac{d \alpha_{r}}{d t} \frac{\sqrt{3}}{2} l \sin \left(\alpha_{r}\right)\right) \mathbf{x}, \alpha_{r} \in\left[\frac{\pi}{3}, \frac{\pi}{2}\right) \& \alpha_{l} \notin\left[\frac{\pi}{3}, \frac{2 \pi}{3}\right) \\
\left(\frac{d \alpha_{r}}{d t} \frac{l}{2} \cos \left(\alpha_{r}\right)+\frac{d \alpha_{r}}{d t} \frac{\sqrt{3}}{2} l \sin \left(\alpha_{r}\right)\right) \mathbf{x}, \alpha_{r} \in\left[\frac{\pi}{2}, \frac{2 \pi}{3}\right) \& \alpha_{l} \notin\left[\frac{\pi}{3}, \frac{2 \pi}{3}\right)
\end{array}\right.
$$

Note that in the Equation (18), the right foot and left foot shares the same function, we compute the left foot angle trajectory for support phase and the right foot angle trajectory will be acquired by applying a time shift.

It is assumed that at $t=0$, the left foot initiates the support phase, and the angle $\alpha$ is increasing with respect to time, the initial condition yields that

$$
\alpha_{i}=\frac{\pi}{3}
$$

The requirement of the robot is to move with a constant forward velocity $v$, thus, the first half of the support phase equations can be written as

$$
-\frac{d \alpha}{d t} \frac{l}{2} \cos (\alpha)+\frac{d \alpha}{d t} \frac{\sqrt{3}}{2} l \sin (\alpha)=v
$$

And for the second half of the support phase, the equation can be written as

$$
\frac{d \alpha}{d t} \frac{l}{2} \cos (\alpha)+\frac{d \alpha}{d t} \frac{\sqrt{3}}{2} l \sin (\alpha)=v
$$

The boundary condition is that at $t=T / 2, \alpha_{i}=2 \pi / 3$. 
Note that $\sin (2 \pi / 3)=\sqrt{3} / 2$ and $\cos (2 \pi / 3)=-0.5$, the equation for the first half of the support phase can then be written as

$$
\cos \left(\alpha-\frac{2 \pi}{3}\right) \frac{d}{d t} \alpha=\frac{v}{l}, \alpha \in\left[\frac{\pi}{3}, \frac{\pi}{2}\right)
$$

And the second half of the support phase as

$$
\cos \left(\alpha+\frac{2 \pi}{3}\right) \frac{d}{d t} \alpha=\frac{v}{l}, \alpha \in\left[\frac{\pi}{2}, \frac{2 \pi}{3}\right)
$$

With reference to Equation (15), the right-hand side of the equations can be computed as

$$
\frac{v}{l}=\frac{2 \sqrt{3}-2}{T}
$$

Integrating both sides of the equation yields

$$
\begin{aligned}
& \alpha=\arcsin \left((2 \sqrt{3}-2) \frac{t}{T}-C_{1}\right)+\frac{2 \pi}{3}, \alpha \in\left[\frac{\pi}{3}, \frac{\pi}{2}\right) \\
& \alpha=\arcsin \left((2 \sqrt{3}-2) \frac{t}{T}-C_{2}\right)+\frac{\pi}{3}, \alpha \in\left[\frac{\pi}{2}, \frac{2 \pi}{3}\right]
\end{aligned}
$$

Here, $C_{1}$ and $C_{2}$ are integral constants which can be obtained by initial conditions.

Applying the initial condition and the boundary conditions, the trajectory of input angle $\alpha$ yields:

$$
\begin{gathered}
\alpha=\arcsin \left((2 \sqrt{3}-2) \frac{t}{T}-\frac{\sqrt{3}}{2}\right)+\frac{2 \pi}{3}, \alpha \in\left[\frac{\pi}{3}, \frac{\pi}{2}\right) \\
\alpha=\arcsin \left((2 \sqrt{3}-2) \frac{t}{T}-\frac{\sqrt{3}}{2}+1\right)+\frac{\pi}{3}, \alpha \in\left[\frac{\pi}{2}, \frac{2 \pi}{3}\right]
\end{gathered}
$$


To achieve a smooth transition from the support phase to swing phase, quintic splines are used to generate the rotation angle trajectory of the Reuleaux triangle in the time domain for a swing phase.

The swing phase trajectory is

$$
\alpha=a_{1}\left(t-\frac{T}{2}\right)^{5}+a_{2}\left(t-\frac{T}{2}\right)^{4}+a_{3}\left(t-\frac{T}{2}\right)^{3}+a_{4}\left(t-\frac{T}{2}\right)^{2}+a_{5}\left(t-\frac{T}{2}\right)+a_{6}
$$

Where $a_{1}, a_{2}, a_{3}, a_{4}, a_{5}, a_{6}$ are calculated as:

$$
\begin{aligned}
& a_{1}=\frac{64(-18+14 \sqrt{3}-5 \pi)}{T^{5}} \\
& a_{2}=\frac{80(-18+14 \sqrt{3}-5 \pi)}{T^{4}} \\
& a_{3}=\frac{16(-102+78 \sqrt{3}-25 \pi)}{T^{3}} \\
& a_{4}=\frac{16(-3+2 \sqrt{3})}{T^{2}} \\
& a_{5}=\frac{4(-1+\sqrt{3})}{T} \\
& a_{6}=\frac{2 \pi}{3}
\end{aligned}
$$

The trajectory profile of the input angle is shown in Figure 4.2.

Equation (23), (24), (25), and (26) demonstrate that the Reuleaux triangle cam trajectory is a function of time $t$ and gait cycle period $T$ only. Let $\tau$ be defined as a non-dimensional value representing the percentage of the gait cycle. The Reuleaux triangle cam trajectory profile is shown in Figure 4.2. Note that in the Figure 4.2, the profile shows the relation of the Reuleuax triangle cam with respect to the percentage of a gait cycle. Hence, the Reuleaux triangle angle trajectory profile is independent from the gait cycle chosen. 


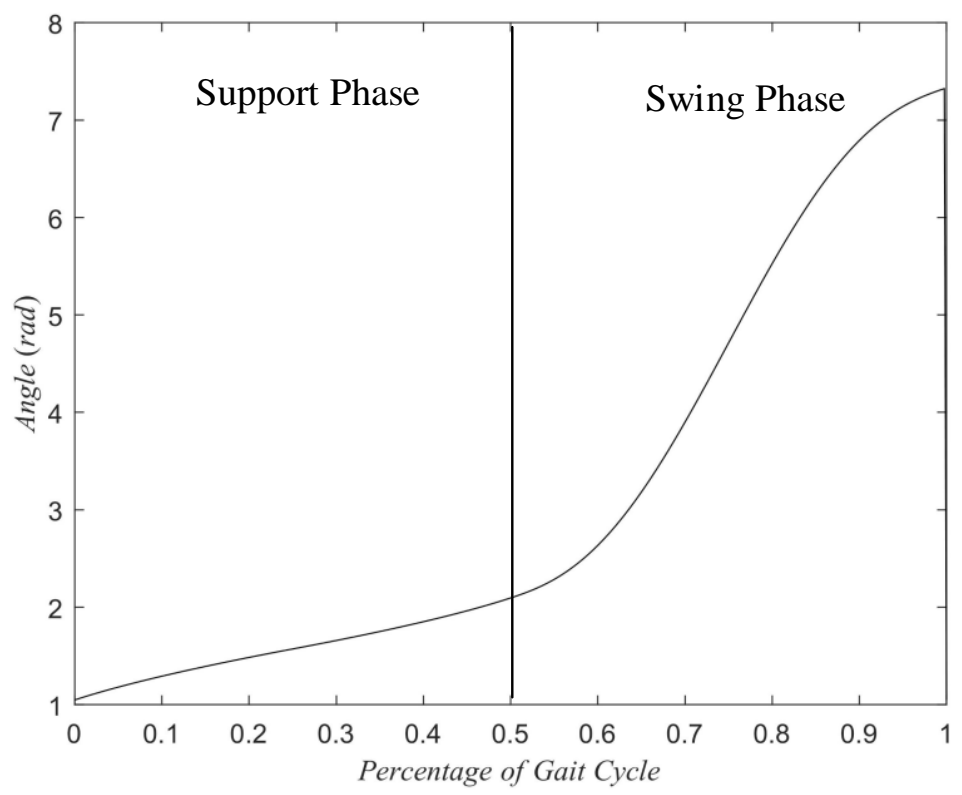

Figure 4.2: Trajectory profile of the input angle $\alpha$

The Reuleaux triangle angular velocity trajectory profiles are shown in Figure 4.3. Note that in Figure 4.3, the maximum angular velocity of the Reuleaux triangle reaches a maximum in a gait cycle when $\tau=3 / 4$ for the left leg or $\tau=1 / 4$ for the right leg. The maximum angular velocity of the Reuleaux triangle cam can be calculated as

$$
\omega_{\text {max cam }}=\frac{13-11 \sqrt{3}+10 \pi}{2 T}
$$

The maximum velocity of the robot is determined by the performance of the motor, therefore, let $\omega_{\max }$ be defined as the maximum velocity the motor can achieve and set $\omega_{\operatorname{maxcam}}=\omega_{\max }$, the minimum gait cycle of the robot can be calculated as

$$
T=\frac{13-11 \sqrt{3}+10 \pi}{2 \omega_{\max }}
$$

Hence, the maximum velocity of the robot yields

$$
v_{\max }=\frac{4(-1+\sqrt{3}) l \omega_{\max }}{13-10 \sqrt{3}+10 \pi}
$$




\section{CHAPTER 5}

\section{DYNAMIC ANALYSIS AND CONTROLS}

\subsection{Kane method}

The dynamic analysis is conducted using Kane's method. Kane's method for dynamic system analysis is getting increasingly popular. It is introduced by Dr. Thomas Kane in his book Dynamics, Theory and Applications in 1985. It offers benefits of both Lagrangian method and Newton-Euler methods. The Kane's method eliminates the contact forces which are workless. And unlike Lagrangian method, Kane's method does not rely on computing the partial derivatives of the Lagrangian, which is a function of the total kinetic and potential energy functions, with respect to each coordinate. Instead, Kane's method relies on matrix multiplications to derive the equation of motion[28].

The procedure of Kane's method involves the following steps:

1. Determine the coordinate frame of different bodies.

2. Develop the angular and translational relations between different coordinate frames.

3. Derive the translational and angular velocity as well as the acceleration of each body in the system.

4. Calculate the generalized inertia force and inertia torque of each body.

5. Calculate the generalized active force and torque on each body.

6. Apply the D'Alembert Principle $\sum F+\sum F^{*}=0$ which yields the equations of motion of the multibody system. 
The equation of motion derived using Kane's method does not require further rearrangement to obtain a form for computer implementation.

\subsection{Dynamic Analysis}

This section presents the dynamic analysis of the biped robot. The coordinate frames of each joint are defined as in Figure 5.1. Figure 5.1 (a) shows the left side view of the robot and Figure 5.1 (b) shows the right view of the robot. The RML-V2 is a planar mechanism, the frames are defined such that the z-axis of each frame is perpendicular to the plan where the mechanism operates, $\mathrm{x}$-axis is always horizontal and $\mathrm{y}$-axis is always vertical.
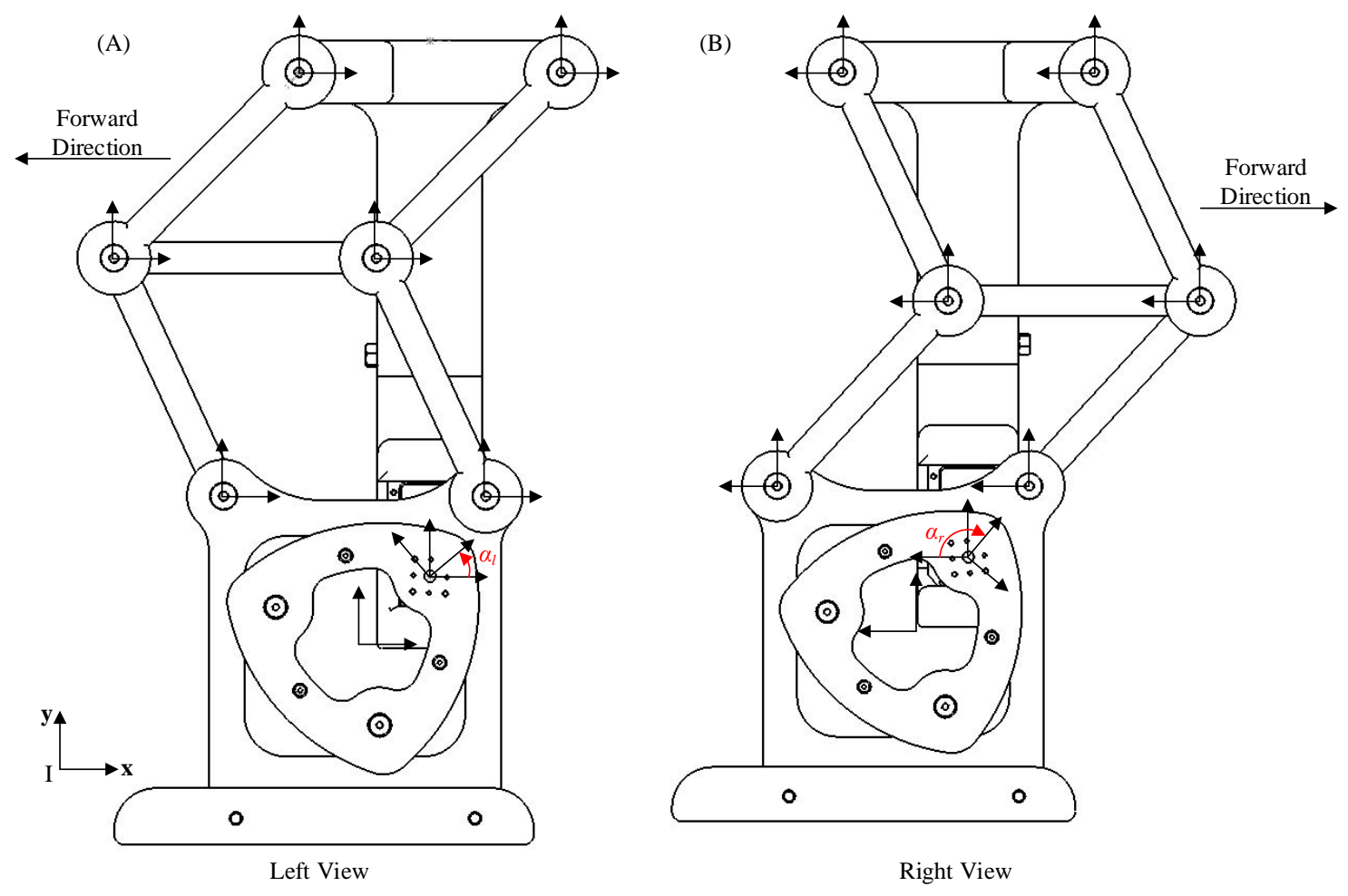

Figure 5.1: The coordinate frame of each joint

In Figure 5.1, the coordinate frames on the left leg and the right leg are provided. To simplify the dynamic analysis of the system, it is assumed that the double four-bar mechanism has negligible mass properties. Thus, only the Generalized Reuleaux triangle cams and their conjugate 
square foot should be considered in the analysis. The relation of the coordinate frames of each link is presented in Table 5.1.

Table 5.1: The Relation of the Coordinate Frames

\begin{tabular}{|ccc|}
\hline & Displacement & Angle \\
\hline World to Body & $(G x, 0,0)$ & \\
\hline Body to LeftMotor & $(0,0,-d m)$ & $\alpha_{l}$ \\
\hline LeftMotor to LeftFoot & $\left(G x\left(\alpha_{l}\right), G y\left(\alpha_{l}\right), 0\right)$ & $-\alpha_{l}$ \\
\hline BeftFoot to LeftFootContact & $(0, l f, 0)$ & \\
\hline RightMotor to RightFoot & $\left(G x\left(\alpha_{r}\right), G y\left(\alpha_{r}\right), 0\right)$ & $-\alpha_{r}$ \\
\hline RightFoot to RightFootContact & $(0, l f, 0)$ & \\
\hline
\end{tabular}

Note that in Table 5.1, the position vector of each individual links can be written as a function of $\alpha_{l}$ or $\alpha_{r}$. Also notice that $\alpha_{l}$ and $\alpha_{r}$ are mutually independent. Therefore, the generalized coordinate can be defined as $\boldsymbol{q}=\left[q_{1}, q_{2}\right]^{T}=\left[\alpha_{l}, \alpha_{r}\right]^{T}$. Hence, the velocity and angular velocity if each link can be calculated as:

$$
\begin{gathered}
\mathbf{v}_{i}=\mathbf{J}_{v i} \dot{\mathbf{q}} \\
\boldsymbol{\omega}_{i}=\mathbf{J}_{\omega i} \dot{\mathbf{q}}
\end{gathered}
$$

Here, $\boldsymbol{J}_{v i}$ is 2 by 3 matrix and $\boldsymbol{J}_{\omega i}$ is 2 by 1 matrix denoted as the Jacobian of $i$-th link. The acceleration of $i$-th link can be computed as

$$
\begin{aligned}
& \mathbf{a}_{i}=\mathbf{J}_{v i} \ddot{\mathbf{q}}+\dot{\mathbf{J}}_{v i} \dot{\mathbf{q}} \\
& \boldsymbol{\beta}_{i}=\mathbf{J}_{\omega i} \ddot{\mathbf{q}}+\dot{\mathbf{J}}_{\omega i} \dot{\mathbf{q}}
\end{aligned}
$$

The inertia force and inertia torque of $i$-th link can then be obtained by 


$$
\begin{aligned}
& \mathbf{F}_{i}^{*}=-m \mathbf{a}_{i}=-m\left(\mathbf{J}_{v i} \ddot{\mathbf{q}}+\dot{\mathbf{J}}_{v i} \dot{\mathbf{q}}\right) \\
& \mathbf{T}_{i}^{*}=-\left(\mathbf{I}_{i} \boldsymbol{\beta}_{i}+\boldsymbol{\omega}_{i} \times \mathbf{I}_{i} \boldsymbol{\omega}_{i}\right)=-\left(\mathbf{I}_{i}\left(\mathbf{J}_{\omega i} \ddot{\mathbf{q}}+\dot{\mathbf{J}}_{\omega i} \dot{\mathbf{q}}\right)+\tilde{\boldsymbol{\omega}}_{i} \mathbf{I}_{i} \boldsymbol{\omega}_{i}\right)
\end{aligned}
$$

Here, $\boldsymbol{a}_{i}, \boldsymbol{\beta}_{i}$ and $\boldsymbol{I}_{i}$ are acceleration, angular acceleration and the inertia matrix of the $i$-th link, respectively. The generalized inertia force of the $i$-th link can be computed by:

$$
\mathbf{K}_{i}^{*}=\mathbf{J}_{v i}^{T} \mathbf{F}_{i}^{*}+\mathbf{J}_{\omega i}^{T} \mathbf{T}_{i}^{*}
$$

Substituting Equation (36) into Equation (37) and rearrange variables, the generalized inertia force yields

$$
\mathbf{K}_{i}^{*}=-\left(\mathbf{J}_{v i}^{T} m_{i} \mathbf{J}_{v i}+\mathbf{J}_{\omega i}^{T} \mathbf{I}_{i} \mathbf{J}_{\omega i}\right) \ddot{\mathbf{q}}-\left(\mathbf{J}_{v i}^{T} m_{i} \dot{\mathbf{J}}_{v i}+\mathbf{J}_{\omega i}^{T} m_{i} \dot{\mathbf{J}}_{\omega i}\right) \dot{\mathbf{q}}-\mathbf{J}_{\omega i}^{T} \tilde{\boldsymbol{\omega}}_{i} \mathbf{I}_{i} \boldsymbol{\omega}_{i}
$$

Here, $\tilde{\boldsymbol{\omega}}_{i}$ is the skew matrix of $\boldsymbol{\omega}_{\mathrm{i}}$. Let $\mathbf{M}_{i}=\mathbf{J}_{v i}^{T} m_{i} \mathbf{J}_{v i}+\mathbf{J}_{\omega i}^{T} \mathbf{I}_{i} \mathbf{J}_{\omega i}$, the equation can be rewritten as

$$
\mathbf{K}_{i}^{*}=-\mathbf{M}_{i} \ddot{\mathbf{q}}-\left(\mathbf{J}_{v i}^{T} m_{i} \dot{\mathbf{J}}_{v i}+\mathbf{J}_{\omega i}^{T} m_{i} \dot{\mathbf{J}}_{\omega i}\right) \dot{\mathbf{q}}-\mathbf{J}_{\omega i}^{T} \tilde{\mathbf{\omega}}_{i} \mathbf{I}_{i} \mathbf{\omega}_{i}
$$

In the equation, $M_{i}$ can be considered as the mass properties viewed from the motor of each link, hence, $-\boldsymbol{M}_{i} \ddot{\boldsymbol{q}}$ is the inertia force that caused by the acceleration of the motor. The term $-\left(\mathbf{J}_{v i}^{T} m_{i} \dot{\mathbf{J}}_{v i}+\mathbf{J}_{\omega i}^{T} m_{i} \dot{\mathbf{J}}_{\omega i}\right) \dot{\mathbf{q}}-\mathbf{J}_{\omega i}^{T} \tilde{\mathbf{\omega}}_{i} \mathbf{I}_{i} \boldsymbol{\omega}_{i}$ represents the Coriolis and the centrifugal force of the $i$-th link.

The external force applied to the body is gravity and input torque $\boldsymbol{\tau}_{l}, \boldsymbol{\tau}_{\mathrm{r}}$ that drive the Reuleaux triangle cam. The generalized external force is calculated as:

$$
\mathbf{K}_{i}=\mathbf{J}_{v i}^{T} m_{i} \mathbf{g}+\mathbf{J}_{\omega i}^{T} \boldsymbol{\tau}_{l}+\mathbf{J}_{\omega i}^{T} \boldsymbol{\tau}_{r}
$$

The equation of motion of the robot is then derived with $\boldsymbol{J}_{C}^{T}$ as the transpose of the constraint Jacobian and $\lambda$ as the Lagrangian multiplier [29].

$$
\sum \mathbf{K}_{i}+\sum \mathbf{K}_{i}^{*}+\mathbf{J}_{C}^{T} \boldsymbol{\lambda}=\mathbf{0}
$$




\subsection{Bipedal Robot Controls}

The robot is controlled based on the angle of each Reuleaux triangle. During locomotion, the controller controls the motors to follow the desired angle trajectory of each generalized Reuelaux triangle cam. A home configuration is selected such that when the robot is powered up, the feet of the robot will automatically move to the home configuration first and start walking. The home configuration is chosen such that it can provide maximum stability i.e. both feet are in contact with the ground. With reference to Chapter $4, \mathbf{q}_{1}=[\pi / 3,2 \pi / 3]^{T}$ or $\mathbf{q}_{2}=[2 \pi / 3, \pi / 3]^{T}$ satisfy the requirement for a home configuration where one foot initiates support phase while the other foot initiates swing phase. When $\mathbf{q}=[\pi / 3,2 \pi / 3]^{T}$, left foot initiates support phase and right foot initiates swing phase and vice versa.

A simple PI controller is used to track the desired angle trajectory. The control method is shown in Figure 5.2

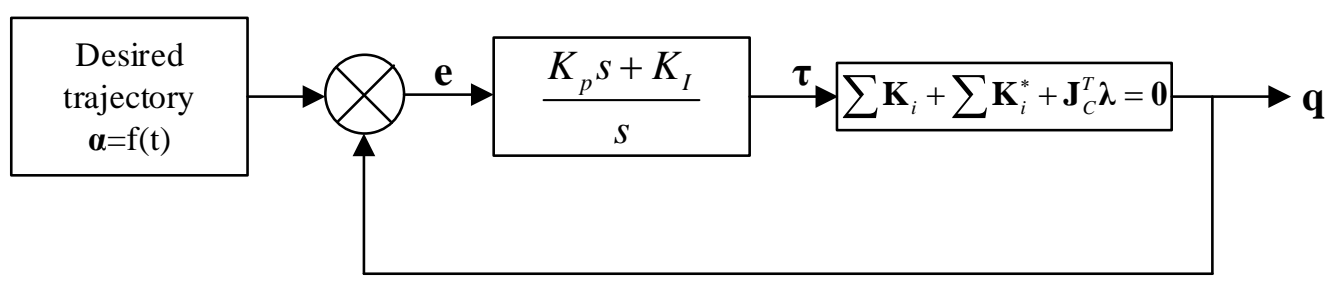

Figure 5.2: The control block diagram of the robot

An 8 second process is simulated in the MATLAB with gait cycle defined as 4 seconds. The result of the left motor angle (A) and right motor angle (B) are presented in Figure 6.3.

In Figure 5.3, the blue dashed line represents the output angle $q$ of the system and the black solid line represents the foot trajectory which the motors should track. In the simulation, the initial configuration is set such that $\mathbf{q}=[\pi / 3,2 \pi / 3]^{T}$ and $\dot{\mathbf{q}}=[\sqrt{3}-1, \sqrt{3}-1]^{T}$. The average tracking 
error of the controller for the left foot is 0.0079 radians with a standard deviation of 0.0085 and the average tracking error of the right foot is 0.0078 radians with a standard deviation of 0.0085 .
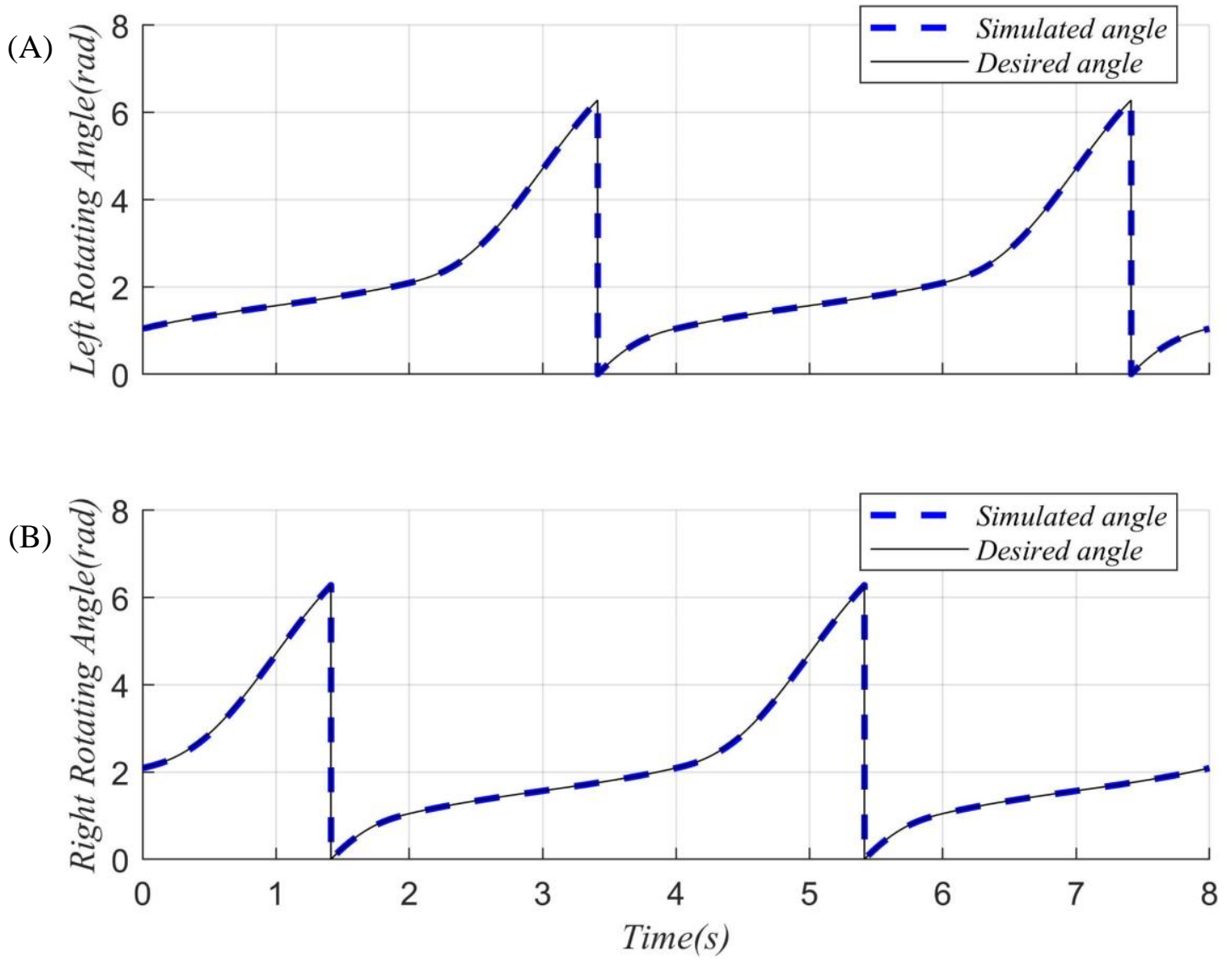

Figure 5.3: Simulated PI controller tracking results

Figure 5.4 shows the torque on the motor used to achieve the trajectory in Figure 5.3. Figure 5.4 shows the torque on the motor used to achieve the trajectory in Figure 5.3. The torque of the motor reaches a maximum during the swing phase of a gait cycle to overcome gravitational and frictional loading of the foot. During the support phase, the motor torque is used to overcome the friction between the Reuleaux triangle and its conjugate square foot. 

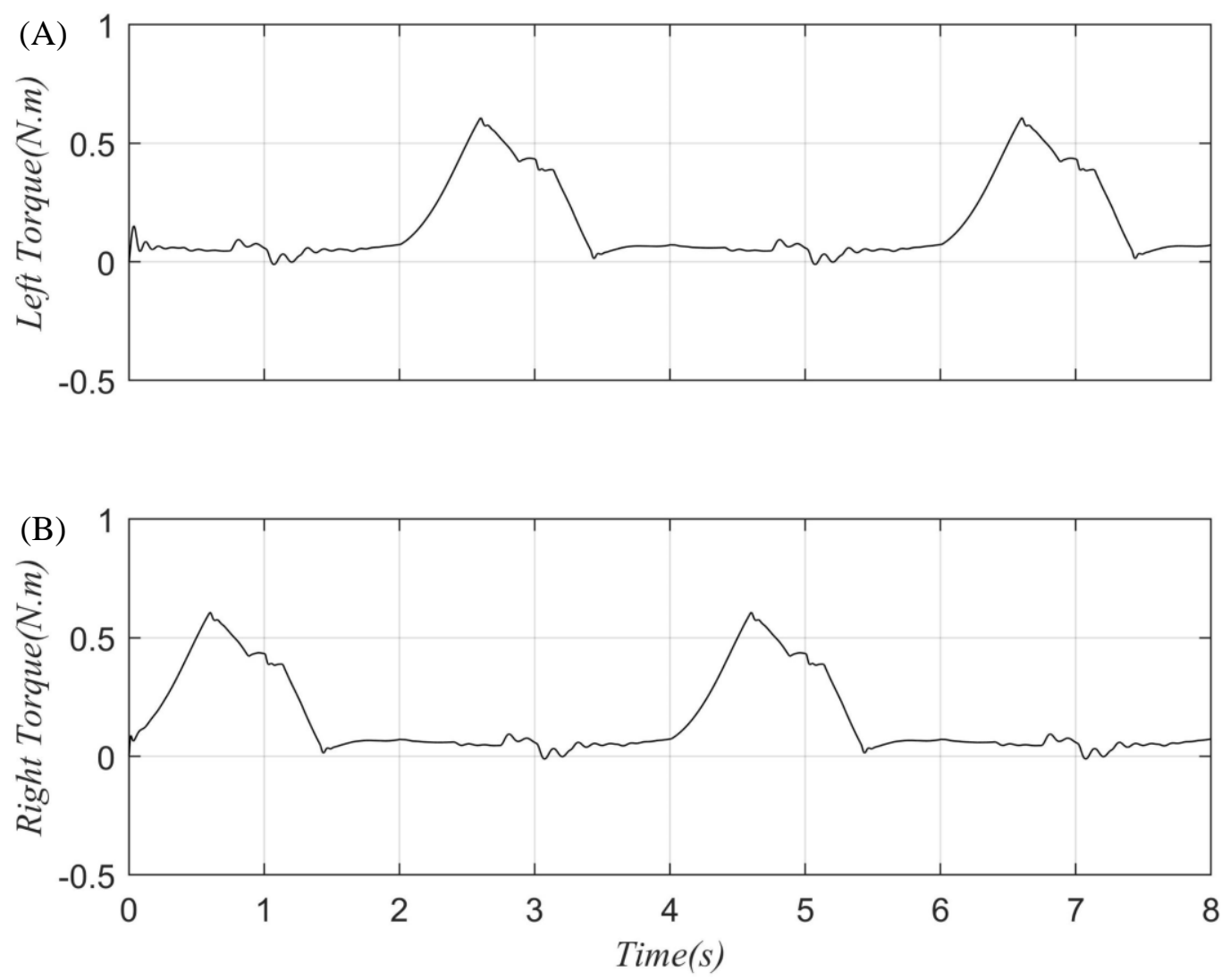

Figure 5.4: Calculated torque on both motors 


\section{CHAPTER 6}

\section{EXPERIMENTAL RESULTS}

A prototype of the robot was built to verify the calculations and control methods. The experiments involved foot trajectory tracking and demonstration of walking capabilities.

\subsection{Prototype}

The prototype measures $230 \times 200 \times 320 \mathrm{~mm}$ and weights $2.5 \mathrm{~kg}$. The Reuleax triangle dimensions were selected to produce a gait cycle with a step height of $75 \mathrm{~mm}$ and a step length of $54.9 \mathrm{~mm}$. The prototype is powered by two Dynamixel MX-106 servo motors which can be controlled based on position and are able to achieve full rotation. The robot is connected to the computer via a USB cable and the motors commands are sent from the computer. Therefore, the prototype does not require any additional microcontrollers. The prototype of the robot is presented in Figure 6.1

The baudrate for the communication between the computer and the motor is $57600 \mathrm{bit} / \mathrm{s}$ second. The sensors integrated into the Dynamixel motors send the absolute motor angle to the computer, which controls the motor to follow the desired Reuleaux triangle cam angle trajectory. Using position feedback, the controller measures the error between the current Reuleaux triangle cam angle and reference angle presented in Chapter 4. The PI controller then outputs the required speed of the motor to follow the desired foot trajectory. The speed information is sent to the Dynamixel. The Dynamixel onboard controller compares the error between the input speed and current motor speed of the motor. The onboard PI controller outputs PWM signal which drives the actual motor which outputs the desired Reuleaux triangle angle trajectory. 


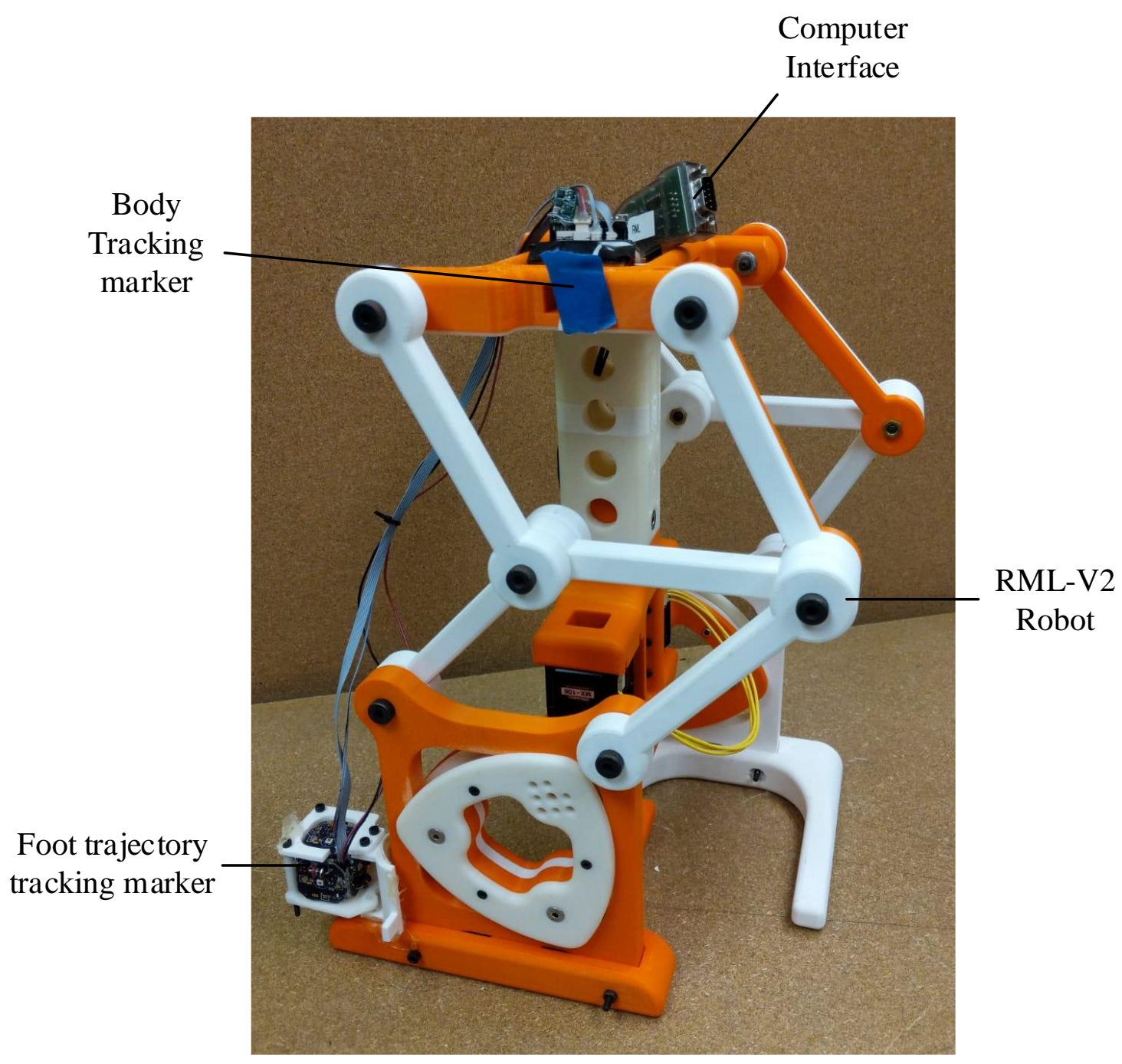

Figure 6.1: The prototype of the biped robot

\subsection{Foot Trajectory}

In this experiment, one motor is set to rotate with a constant angular velocity while the other remain still to provide support of the robot. The foot of the moving leg was removed from its foot assembly for this experiment to avoid motion interference between the floor and the other foot. Linear Optical Sensor Arrays (LOSA) [30] tracking system is used to track the foot trajectory with respect to the ground. 
The foot trajectory tracking experiment compares the theoretical trajectory. The foot of the foot assembly was removed to eliminate potential motion interference. The prototype was fixed to a stable surface via a c-clamp. A marker was fixed on the foot conjugate square box and was tracked by a computer vision method. In this experiment, one of the Reuleaux triangle cam was set to rotate at a constant velocity while the other remained stationary; tracking results versus theoretical results are presented in Figure 6.2. The actual foot trajectory is presented as a dashed line and the theoretical trajectory is presented as a solid line.

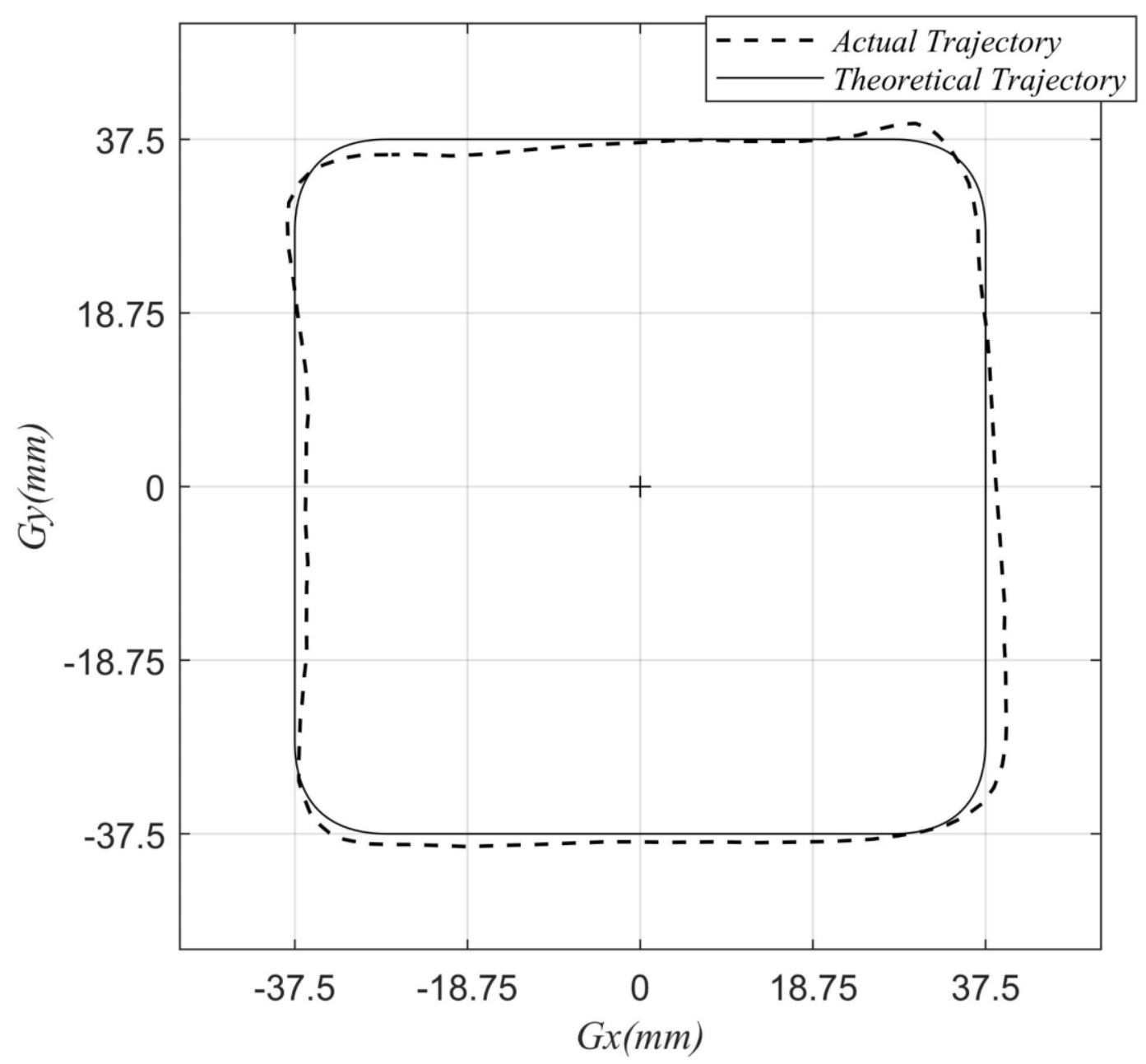

Figure 6.2: Experimental and calculated foot trajectory 

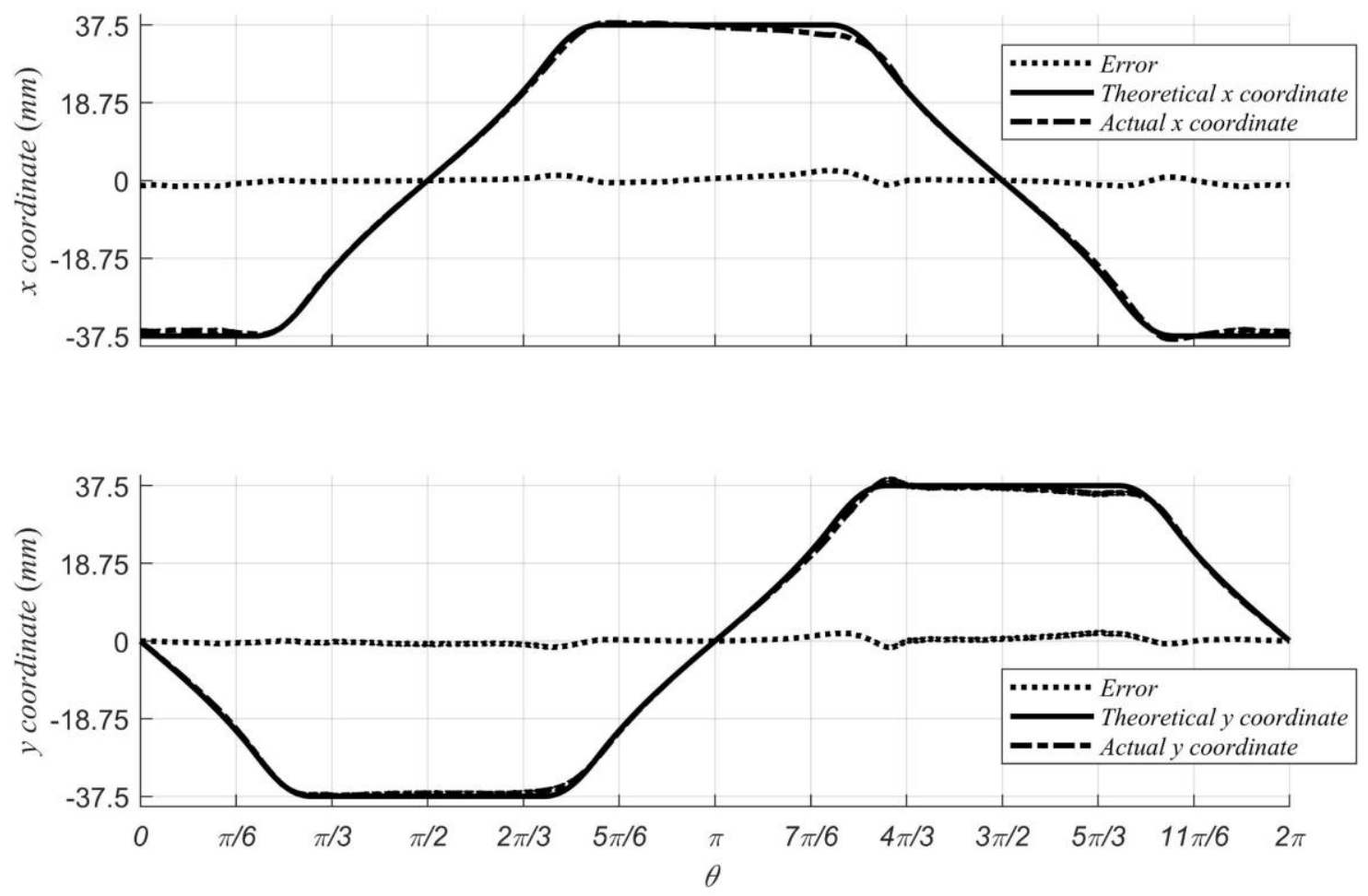

Figure 6.3: $x$ error and $y$ error of experimental results versus theoretical results

Let $\theta=\operatorname{atan} 2(G x / G y)$ represent the angular displacement of the foot in polar coordinate. Figure 6.3 presents the tracking error in $x$ and $y$ with respect to $\theta$, along with the theoretical trajectories of $x$ and $y$ and for the foot's rotation cycle. In Figure 6.3, the theoretical trajectory is presented as a solid line, experiment trajectory is presented as a dash-dot line and the trajectory of errors between theoretical trajectory and the experimental trajectory is presented as a dotted line.

With reference to Figure 6.4, the overall average error of $x$-coordinate is 0 with a standard deviation of 0.86 and the overall average error of $y$-coordinate is 0.03 with a standard deviation of 0.82. Note that during the support phase, the $y$-coordinate of the theoretical result and the experimental result shows a small error. Further analysis indicates that the average $y$-coordinate error during the support phase is -0.61 (1.63 percent) with a standard deviation of 0.11 . Therefore, it can be safely assumed that the actual support phase of the prototype is a straight line. 


\subsection{Walking Capability}

This section tested the robot walking performance on flat floors (6.3.1), rough terrains (6.3.2) and its stair climbing capabilities (6.3.3). The flat floor walking performance evaluated the robot walking performances in the lab environment to test if the robot satisfies the design requirement. The rough terrain walking test and stair climbing test was used as additional test to check the robots capabilities of other type of terrains, and experimental results of additional tests were used as a reference for future researches.

\subsubsection{Walking performance on flat floor}

The walking capabilities experiment evaluated the prototype's performance during walking. The experiment contained two sections, (a) forward walking and (b) steering. In the forward walking section, the robot was commanded to walk forward, and in the steering section, one foot was set stationary while the other foot remains operating. The velocity of the robot and the body height fluctuation was evaluated for the forward walking section of the experiment and the steering capability is evaluated in the steering section of the robot.

The input angle trajectory and gait sequence discussed in Chapter 4 were used in the forward walking experiment. The experiment set up is shown in Figure 6.4.

Computer vision method [31] used for actual foot trajectory tracking was used to track the body movement. As shown in Figure 6.4, in this experiment, the prototype was put on a solid surface without restriction such that the prototype could move freely. The gait cycle was set to five seconds for the forward walking capability test. A tracker was placed on the body of the robot such that the displacement of the robot body could be acquired via the computer vision method. 

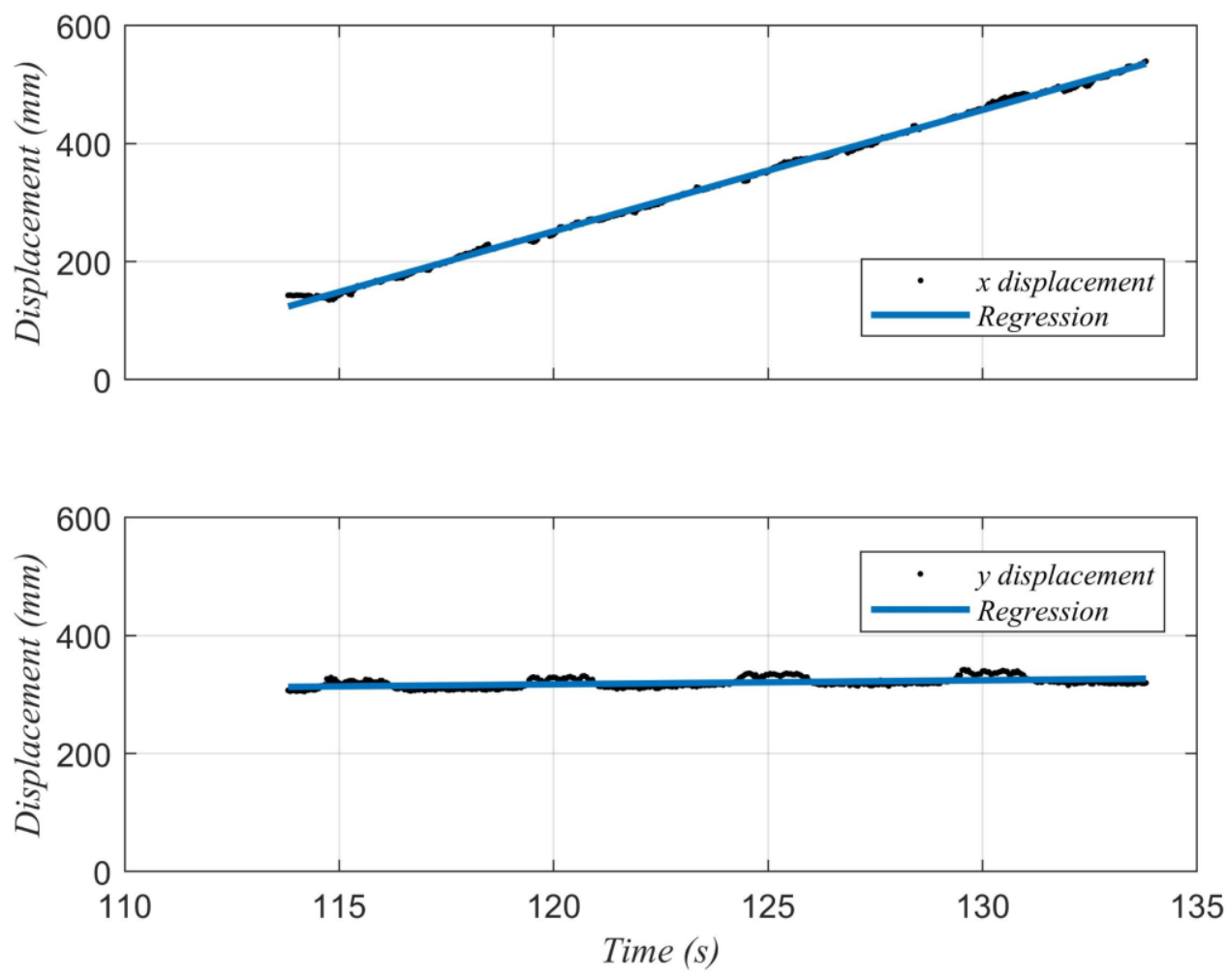

Figure 6.4: Straight walking prototype body $x$ and $y$ displacement

The initial configuration was set to be $\mathbf{q}=[\pi / 3,2 \pi / 3]^{T}$. In the experiment, the motors moved to the initial configuration, then the motor rotated based on the foot trajectory obtained in Chapter 4. According to Equation (15), a 5-second gait cycle yields the theoretical forward velocity of the robot to be $21.9 \mathrm{~mm} / \mathrm{s}$. The tracking results of the $x$ and $y$ displacement with respect to time is presented in Figure 6.4.

The $x$-displacement data shows the information about the walking velocity and $y$-displacement shows the body height fluctuation during walking. Note that in Figure 6.4, the $x$-displacement demonstrates a linear pattern with respect to time. This indicates that the robot moves with a constant velocity and the slope of the $x$-displacement is the forward velocity of the prototype. 
The linear regression yields

$$
\begin{aligned}
& x=21 t+2200 \\
& R=0.99
\end{aligned}
$$

Therefore, it can be said that the robot is moving with a constant velocity. Based on Equation (35), it is evident the velocity of the prototype is $21 \mathrm{~mm} / \mathrm{s}$, which matches the theoretical results. Thus, the prototype satisfies the criteria of constant velocity. The $y$-displacement shows the body height during walking. Regression techniques yields

$$
y=0.66 t+2300
$$

Note that in Figure 6.4, the body height demonstrates a periodical fluctuation. During the walking, when one foot is in the swing phase while the other is in support phase, the robot has a tendency to roll. The design of the foot, however, ensures that the zero moment point falls in the support polygon. The mass of the body generated a torque at the box-foot connection, which resulted in a roll displacement of the body. During the transition between different phases, two feet are in contact with the ground, therefore, no torque is exerted at the box-foot connection. Therefore, a fluctuation occurred during the walking. However, as noticed in Figure 6.5, the fluctuation of the robot is negligible such that it can still be assumed that the criteria of constant body height during walking is satisfied. 

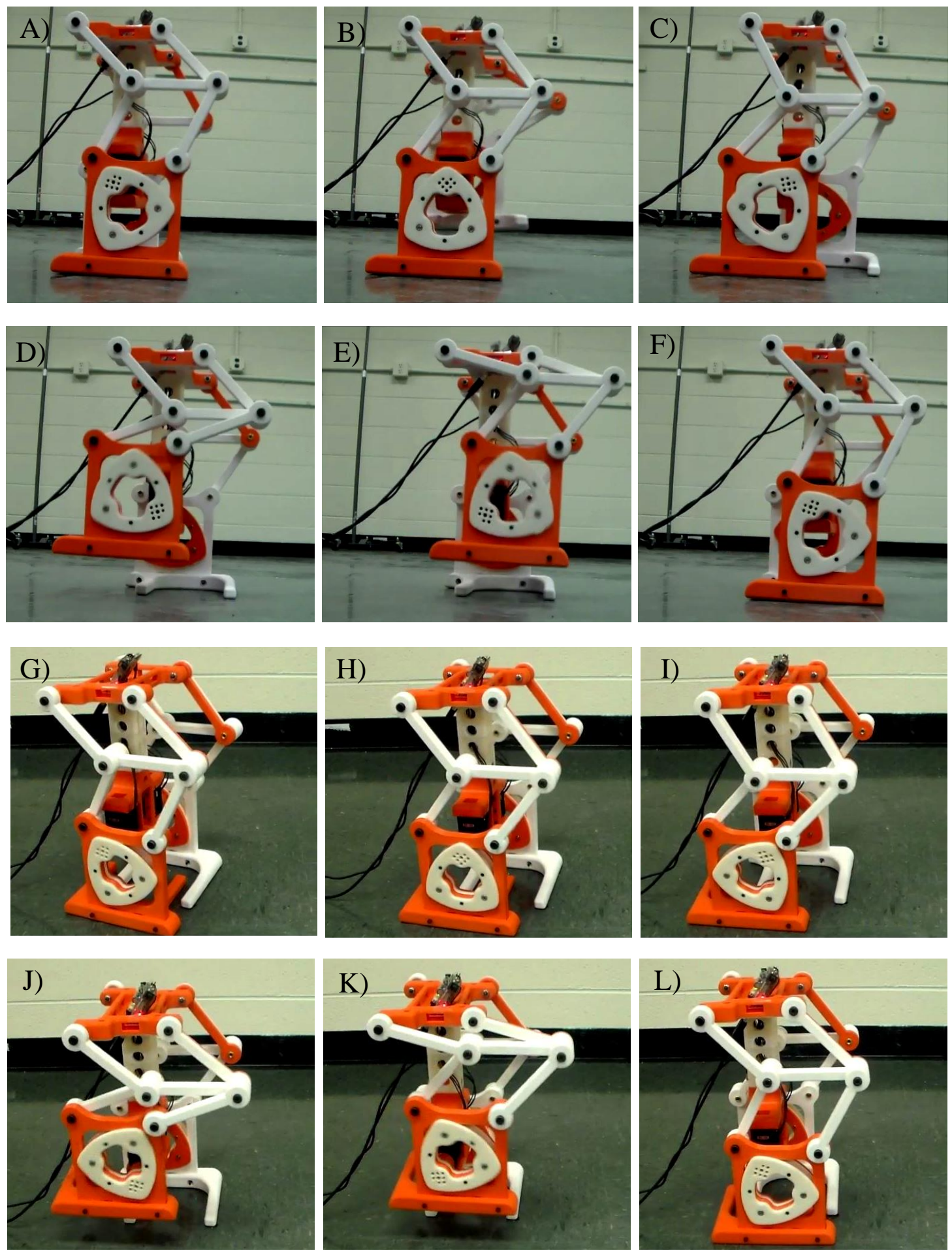

Figure 6.5: Biped robot demonstrating forward locomotion (A-F), and differential turning (G-L) 
As demonstrated in these experiments, the biped robot, which is composed of two single-DOF RML-V2 mechanisms, is capable of satisfying all the walking criteria discussed in Chapter 2, the robot is capable of maintaining quasi-static stability during locomotion, maintaining a constant robot body height and a constant body orientation during a walking gait, all while actively moving forward and steering. For turning capabilities, each individual gait cycle results in $18^{\circ}$ change in direction. While the first generation leg mechanism required two active DOF per leg $[10,11]$, the RML-V2 can produce a forward walking gait approximately three times faster due to the continuous rotation of the Reuleaux triangle and does not require any motor direction changes or synchronization of control effort for producing a valid foot trajectory.

\subsubsection{Walking performance on rough terrains}

This section evaluates the walking performance of the robot on flat rough terrains. Unlike flat floors, which ensure a surface contact between the foot and the floor, these rough terrains provide multiple point contacts between the foot and the ground. Therefore, the stability of the robot walking on rough terrains is hindered and experiments are required to evaluate the stability of the robot during walking on rough terrains.

For this experiment, gravel is chosen as the rough terrain. The experiment set up is shown in Figure 6.6. Marbles were placed evenly on the floor to form a gravel terrain with width $305 \mathrm{~mm}$ and length $915 \mathrm{~mm}$. The marbles formed a rough flat surface which allowed the robot to stand with both feet on the ground.

The robot was then commanded to walk with a gait cycle of 5 seconds. Although the robot demonstrated a stable walking capability with constant body height and body orientation on a flat floor, when one of the foot initiated the swing phase and left the ground, the robot tilted and fells to the ground. By changing the initial location of the robot on the gravel terrains, the robot was 
able to maintain standing with one foot in swing phase and the other in support phase, however, as the robot moved forward, stable walking could not be achieved and the robot fell to the ground. The support polygon of the robot on rough terrains is formed by multiple point contacts between the foot and the ground, therefore, the shape of the support polygon is random, and therefore, the COM of the robot may not be able to fall inside the support polygon generated.

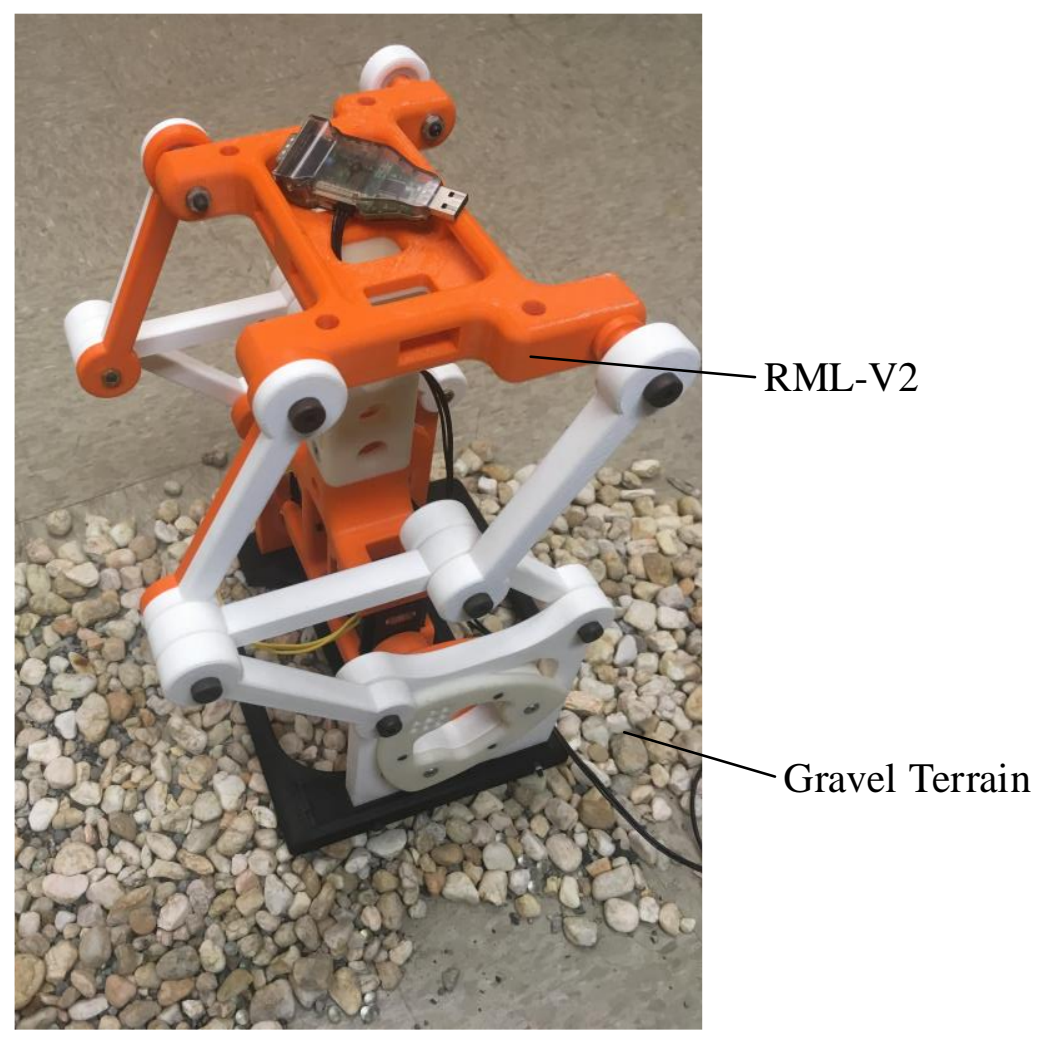

Figure 6.6: Experiment setup for rough terrain walking performance test

\subsubsection{Stair climbing test}

This section evaluates the robot capabilities of climbing stairs. A stair was made with multiple layers of foams with a height measured of $59 \mathrm{~mm}$. In this experiment, the robot was commanded to walk forward with given foot trajectory mentioned in the Chapter 4. Figure 6.7 shows the 
scenario that one foot of the robot touched the stair while the other was in contact with the ground. The experiment shows that the robot fell to the ground when the robot lift its body. The robot configuration in Figure 6.7 also appears in the walking forward experiment when one foot was in swing phase while the other was in support phase. The stable walking on the flat surface indicated that the COM of the robot fell into the support polygon generated by the foot which was in contact with the ground. When climbing the stairs, although the forward foot was in contact with the stairs, the COM of the robot still fell in the support polygon generated by the rear foot, instead of in the polygon on the stairs. Therefore, the robot failed to climb the stairs.

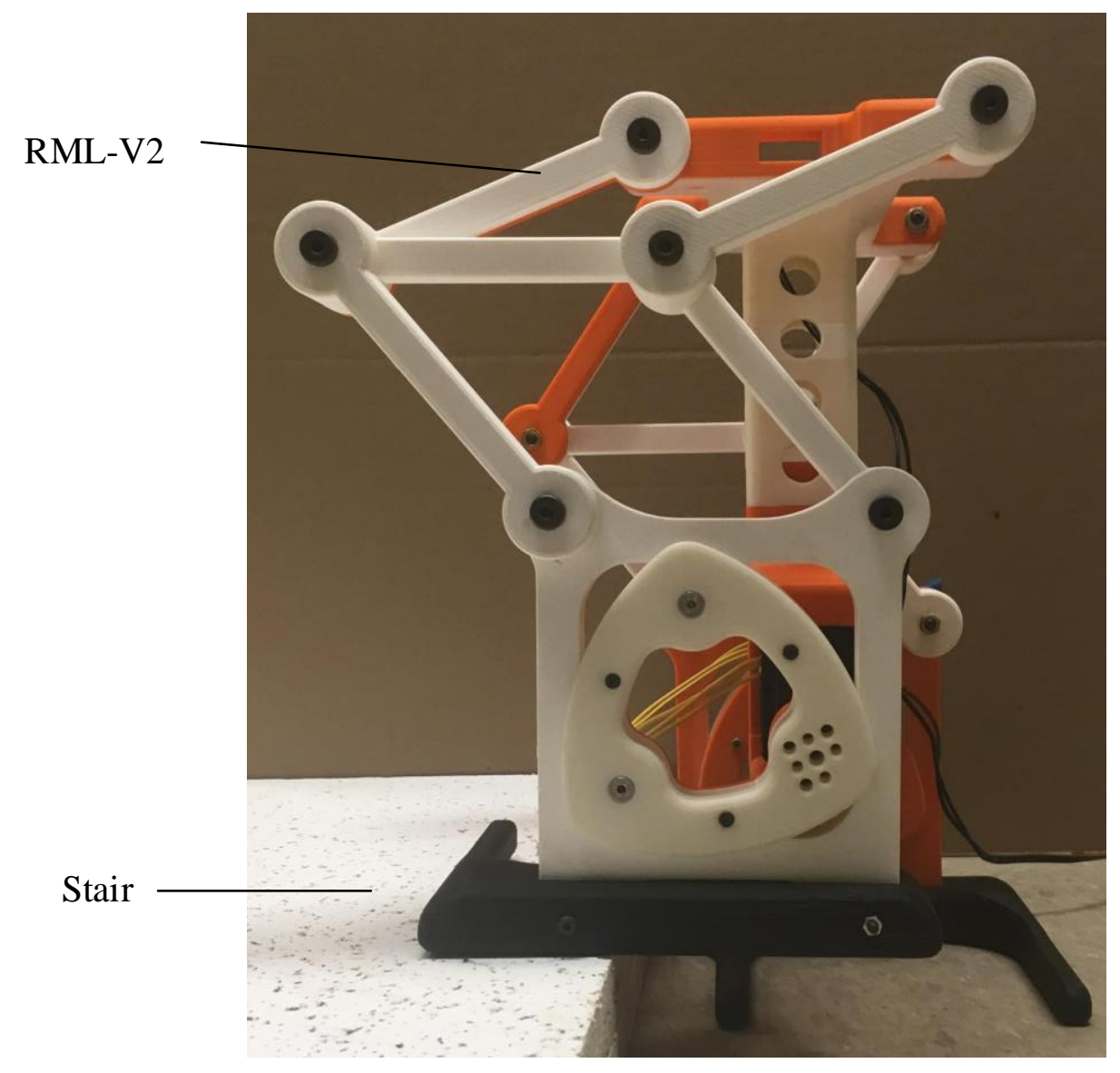

Figure 6.7: Stair climbing experiment 


\section{CHAPTER 7}

\section{CONCLUSION AND FUTURE WORK}

\subsection{Conclusion}

This thesis covered the kinematic analysis of the Reuleaux triangle cam-follower mechanism, the mechanical design, dynamic analysis, prototype integration and the experimental validation of the RML-V2. The proposed design is a walking robot using a single DOF leg mechanism to achieve a bipedal locomotion. The system is capable of maintaining a quasi-static stability during locomotion, maintaining constant body height during walking, maintaining constant velocity during walking, moving forward and steering.

A comprehensive literature review on reduced DOF legs was provided. The review first highlighted the challenges of the conventional robotic legs which relied on many active DOF to enhance locomotion and tasking capabilities. The review then summarized the reduced DOF leg mechanism and robots proposed by other researchers.

The kinematics of the Reuleaux triangle was provided as the preliminary analysis of the leg mechanism. The kinematic behavior of the conjugate foot displayed multiple trajectories profiles which can be used as foot trajectory of the leg mechanism. The requirement for the biped robot was determined and the trajectory profile was chosen to meet the requirement. An optimization procedure was performed to obtain the optimal joint angle trajectory such that the robot could maintain a constant body height and forward velocity during locomotion.

The contribution of this work can be summarized as follows:

1. Proposed a leg mechanism that utilizes the Reuleaux triangle cam-follower system to actuate the robotic legs as a single DOF. Contrary to the prior applications which are 
concern with the motions of the Reuleaux triangle that rotates inside a fixed conjugate square, this application utilizes the rotation of the Reuleaux triangle about a fixed distance from its centroid to generate a translational motion of the conjugate square.

2. Developed a trajectory function of the conjugate square with respect to the offset value from the Reuleaux triangle centroid. The work on the Reuleaux triangle focused on the conjugate square and optimizations of the Reuleaux triangle such that a square trajectory can be achieved. It provided qualitative results of the trajectory profile. This work provided a function of the trajectory with respect to different points along the symmetry axis of the Reuleaux triangle.

\subsection{Future Work}

The robot showed flaws in walking on rough terrains and climbing stairs. Future work involves addressing these flaws by attaching robotic tail mechanism onto the body to increase the stability of the robot on rough terrains and stair climbing. The robotic tail mechanism will be used to adjust the COM of the robot such that it could fall into the support polygon and provide assistance in maneuvering and disturbance rejection [32].

The future work can be detailed as follows:

1. Develop the dynamic model of the tail-robot system.

2. Develop a control law that actively adjust the COM of the robot such that it falls in the support polygon of the robot and assist maneuvering of the robot

3. Experiment validation of the system

Work on the robotic tail is currently being done at Virginia Tech's Robotics and Mechatronics Lab [33]. 


\section{REFERENCE}

[1] S. Feng, E. Whitman, X. Xinjilefu, and C. G. Atkeson, "Optimization based full body control for the atlas robot," in Humanoid Robots (Humanoids), 2014 14th IEEE-RAS International Conference on, 2014, pp. 120-127: IEEE.

[2] M. F. Silva and J. A. T. Machado, "A Historical Perspective of Legged Robots," Journal of Vibration and Control, vol. 13, no. 9-10, pp. 1447-1486, 2007.

[3] J. A. Galvez, J. Estremera, and P. Gonzalez de Santos, "A new legged-robot configuration for research in force distribution," Mechatronics, vol. 13, no. 8, pp. 907932, October 2003.

[4] M. Hutter et al., "Anymal-a highly mobile and dynamic quadrupedal robot," in International Conference on Intelligent Robots and Systems Daejeon, Korea, 2016, pp. 38-44: IEEE/RSJ.

[5] K. Waldron and R. McGhee, "The adaptive suspension vehicle," IEEE Control Systems Magazine, vol. 6, no. 6, pp. 7-12, 1986.

[6] M. Kaneko, M. Abe, K. Tanie, S. Tachi, and S. Nishizawa, "Basic experiments on a hexapod walking machine (MELWALK-III) with an approximate straight-line link mechanism," in International Conference on Advanced Robotics (IEEE), St. Louis, MO, 1985, pp. 397-410.

[7] G. M. Nelson and R. D. Quinn, "Posture control of a cockroach-like robot," in International Conference on Robotics and Automation, 1998, vol. 1, pp. 157-162 vol.1.

[8] A. Schwab and M. Wisse, "Basin of attraction of the simplest walking model," in Proceedings of the ASME design engineering technical conference, 2001, vol. 6, pp. 531539. 
[9] M. Kaneko, M. Abe, S. Tachi, S. Nishizawa, K. Tanie, and K. Komoriya, "Legged locomotion machine based on the consideration of degrees of freedom," in Theory and Practice of Robots and Manipulators: Springer, 1985, pp. 403-410.

[10] W. Saab and P. Ben-Tzvi, "Design and Analysis of a Robotic Modular Leg Mechanism," in International Design Engineering Technical Conferences and Computers and Information in Engineering Conference, Charlotte, NC, 2016: ASME.

[11] W. Saab, W. S. Rone, and P. Ben-Tzvi, "Robotic Modular Leg: Design, Analysis, and Experimentation," Journal of Mechanisms and Robotics, vol. 9, no. 2, pp. 024501024501-6, 2017.

[12] A. Torige, M. Noguchi, and N. Ishizawa, "Centipede type multi-legged walking robot," in Intelligent Robots and Systems' 93, IROS'93. Proceedings of the 1993 IEEE/RSJ International Conference on, 1993, vol. 1, pp. 567-571: IEEE.

[13] K. L. Hoffman and R. J. Wood, "Passive undulatory gaits enhance walking in a myriapod millirobot," in Intelligent Robots and Systems (IROS), 2011 IEEE/RSJ International Conference on, 2011, pp. 1479-1486: IEEE.

[14] H. Adachi, N. Koyachi, and E. Nakano, "Mechanism and control of a quadruped walking robot," IEEE Control Systems Magazine, vol. 8, no. 5, pp. 14-19, 1988.

[15] E. C. Lovasz, C. Pop, F. Pop, and V. Dolga, "Novel solution for leg motion with 5-link belt mechanism," Int. J. Appl. Mech. Eng, vol. 19, no. 4, pp. 699-708, 2014.

[16] L. Patnaik and L. Umanand, "Kinematics and dynamics of Jansen leg mechanism: A bond graph approach," (in English), Simulation Modelling Practice and Theory, vol. 60, pp. 160-169, Jan 2016. 
[17] U. Saranli, M. Buehler, and D. E. Koditschek, "RHex: A simple and highly mobile hexapod robot," The International Journal of Robotics Research, vol. 20, no. 7, pp. 616$631,2001$.

[18] H. Funabashi, K. Ogawa, Y. Gotoh, and F. Kojima, "Synthesis of Leg-Mechanisms of Biped Walking Machines : Part I, Synthesis of Ankle-Path-Generator," Bulletin of JSME, vol. 28 , no. 237 , pp. $537-543,1985$.

[19] C. Tavolieri, E. Ottaviano, M. Ceccarelli, and A. Rienzo, "Analysis and design of a 1DOF leg for walking machines," in 15th International Workshop on Robotics in AlpeAdria-Danube Region (RAAD), Balatonfured, Hungary, 2006, pp. 15-17.

[20] N. Lokhande and V. Emche, "Mechanical spider by using Klann mechanism," International Journal of Mechanical Engineering and Computer Applications, vol. 1, no. 5, pp. 013-016, 2013.

[21] V. R. Kamidi, W. Saab, and P. Ben-Tzvi, "Design and analysis of a novel planar robotic leg for high-speed locomotion," in Intelligent Robots and Systems (IROS), 2017 IEEE/RSJ International Conference on, 2017, pp. 6343-6348: IEEE.

[22] C. Liang, M. Ceccarelli, and Y. Takeda, "Operation analysis of a one-DOF pantograph leg mechanisms," in 17th International Workshop on Robotics in Alpe-Adria-Danube Region (RAAD), Ancona, Italy, 2008, vol. 8, pp. 1-10.

[23] K. Yoneda, Y. OTA, F. Ito, and S. Hirose, "Construction of a quadruped with reduced degrees of freedom," in Industrial Electronics Society, Nagoya, Japan, 2000, vol. 1, pp. 28-33, Nagoya, Japan: IEEE, 2000.

[24] F. Reuleaux and E. S. Ferguson, Kinematics of machinery: outlines of a theory of machines. Courier Corporation, 2012. 
[25] H. J. Watts, "Drill or boring member," United States Patent 1241176, Sep 25, 1917.

[26] G. Figliolini, P. Rea, and S. Grande, "Higher-Pair Reuleaux-Triangle in Square and its Derived Mechanisms," in International Design Engineering Technical Conferences and Computers and Information in Engineering Conference, Chicago, IL, 2012, pp. 16291638: ASME.

[27] S. Wen and P. Huang, Principles of Tribology. John Wiley \& Sons, 2012.

[28] Z. Hussain and N. Z. Azlan, "KANE's method for dynamic modeling," in 2016 IEEE International Conference on Automatic Control and Intelligent Systems (I2CACIS), 2016, pp. 174-179.

[29] J. Wang, V. R. Kamidi, and P. Ben-Tzvi, "A Multibody Toolbox for Hybrid Dynamic System Modeling Based on Nonholonomic Symbolic Formalism," in ASME 2018 Dynamic Systems and Control Conf. (DSCC 2018), Atlanta, GA, 2018: ASME.

[30] A. Kumar and P. Ben-Tzvi, "Spatial Object Tracking System Based on Linear Optical Sensor Arrays," IEEE Sensors Journal, vol. 16, no. 22, pp. 7933-7940, 2016.

[31] S. S. Sohal, W. Saab, and P. Ben-Tzvi, "Improved Alignment Estimation for Autonomous Docking of Mobile Robots," in International Design Engineering Technical Conferences and Computers and Information in Engineering Conference, Quebec City, Canada, 2018, p. V05AT07A072: ASME.

[32] W. S. Rone, Y. Liu, and P. Ben-Tzvi, "Maneuvering and stabilization control of a bipedal robot with a universal-spatial robotic tail," Bioinspiration \& Biomimetics, vol. 14, no. 1, p. $016014,2019$.

[33] Y. Liu and P. Ben-Tzvi, "Dynamic Modeling of a Quadruped With a Robotic Tail Using Virtual Work Principle," in International Design Engineering Technical Conferences and 
Computers and Information in Engineering Conference, Quebec City, Canada, 2018:

ASME. 